CERN LIBRARIES, GENEVA

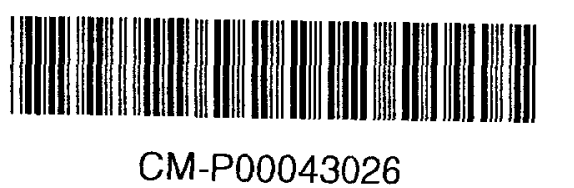

CHS-39

December 1993

CM-P00043026

STUDIES IN CERN HISTORY

PHYSICS IN THE CERN THEORY DIVISION

John ILIOPOULOS

GENEVA

1993 
The Study of CERN History is a project financed by institutions in several CERN Member States.

This report presents preliminary findings, and is intended for incorporation into a more comprehensive study of CERN's history. It is distributed primarily to historians and scientists to provoke discussion, and NO PART OF IT SHOULD BE CITED OR REPRODUCED WITHOUT THE WRITTEN PERMISSION OF THE AUTHOR. Comments and criticism are welcome, and should be sent to the author at

Ecole Normale Supérieure

24 rue Lhomond

F-75231 Paris Cedex 05.

Copyright History of CERN Project, Geneva, 1993 
Physics in the CERN Theory Division

John ILIOPOULOS 


\title{
Physics in the CERN Theory Division
}

\author{
J. Iliopoulos
}

\section{Contents}

1. Introduction

2. Birth of the Group

3. The Copenhagen years

4. Moving to Geneva

5. CERN, the Center of Europe

6. The rise of the Standard Model

7. Beyond the Standard Model

8. Conclusions

References 


\section{Physics in the CERN Theory Division}

\section{Introduction}

This chapter has not been written by a historian. In fact, my respect and my sympathy for historians have considerably increased in the course of this work; so has also my determination never to become one. I fully enjoyed the long hours I spent in the CERN archives reading old documents and manuscript notes, as well as the discussions I had with some of my senior colleagues, but even now, I cannot say that I really know what happened. I know several stories but I cannot reconstruct History. I think that, for the first time, I understood the wisdom of the old movie by Kurosawa 'Rashomon' which I full-heartedly recommend to everybody who wishes to study History. I discovered experimentally that human memory, including my own, is partial and selective and the more certain one feels about his recollection of a particular event, the more one should doubt about it. On the other hand, for having taken part in many committees, I know too well that the entire story rarely appears in the proceedings. Many a time, during my search, I decided, out of frustration, to drop the whole enterprise and it is only because I was reluctant not to honor my promise that I continued. However, I had to compromise. When I started, I intended to write a well-documented historical chapter, solidly supported by archives and witnesses. Yes, I was that naïve! I thought that the matter would have been relatively easy since the facts are recent, I have taken part personally in a large part of them and for the others, I could rely on my contacts with the protagonists, most of whom are still active. This turned out to be an impossible task. An experienced historian, may have been able to find his way through, and solve, the various puzzles of conflicting evidence, but I could not. So this chapter, rather than being the truth, is just my version of the facts. I apologize to those who will find that my story does not correspond to the one they told me. It is not that I was not listening to one of them, but that I was listening to them all. Finally, I had to renounce to answer some of the more 'historical' questions I had asked myself. This chapter, will not tell the story of the CERN Theory Division, it will give instead my idea of the Physics that was done there. Sailing close to the shore of impersonal scientific facts has been my way to avoid being lost in endless speculations. It was an easy solution and, in fact, a rewarding one. For, during these years, we have witnessed one of the greatest triumphs of abstract theoretical thought.

One often says that progress in Physics occurs when an unexpected experimental result contradicts the current theoretical beliefs. This forces physicists to look for new ideas and eventually it gives rise to a New Theory. Such has been the case most of the time in the past, but the revolution that brought Geometry under the form of Gauge Theories into Physics had a theoretical, better an aesthetic, motivation. It is not possible to tell the story of the CERN Theory 
Division without, at the same time, telling the story of this exciting achievement. This is the rewarding part.

\section{Birth of the Group}

The establishment of CERN, its origins as well as the motivations that were behind it, have been presented more expertly and more thoroughly in the first volume of this series. There we find that the first fully operating research group that was created was the Theory Group. (There were no Divisions yet). It may sound curious that one of the first acts of the founding fathers towards an experimental High Energy Center was to hire some theorists and indeed, in this respect, Europe departed from the American model. Brookhaven, the analogue and rival to CERN in these early years, never developed a large theoretical group. What was even more important was that the special role of the theorists in an experimental center was clearly recognized. Although they were actively encouraged to act as consultants to the experimentalists and to participate in the establishment of the experimental programme, this has never been made a necessary condition. Excellence in research was supposed to be the first criterion. In fact, we can find among the early fellows, as well as among the staff members later, several theorists whose interests were abstract and mathematical.

I do not know for sure who first suggested the establishment of a group of theoretical studies, but I suspect that its spiritual father was no lesser man than Niels Bohr, an already legendary figure in Theoretical Physics. Since the early days of Quantum Theory, Niels Bohr had run in Copenhagen the most famous and the most successful Institute of Theoretical Physics of all times. Copenhagen became intimately connected with Quantum Mechanics. The Niels Bohr Institute was the Mecca (or Jerusalem), the place where, for generations, every young theorist aspired to go. But times had changed. The Old Master was more and more absorbed in his activities for disarmament and prevention of nuclear war. Europe, divided between East and West, had not yet healed the wounds of the war. Supremacy in scientific research had crossed the Atlantic and found new homes in the flourishing American Universities. The newly created European Organization for Nuclear Research offered to Bohr an opportunity to revive his Institute. In a historical decision the Interim Council of CERN, in its first session in May 1952 in Paris, decided to create a Group of Theoretical Studies and to appoint Niels Bohr at its head. The group would be provisionally hosted by the Institute in Copenhagen.

\section{The Copenhagen years}

What this decision meant in practice was that CERN funds were available to hire theorists from the member states in Copenhagen. Indeed, for the year 1952-53 we find the first fellows: 


\section{THEORETICAL GROUP STAFF (COPENHAGEN) AS AT SEPTEMBER 1954}

Names
N. Bohr
A.O.G. Källen

E. Abrahamsen
L. Michel
J.E. Hooper
T. Sigurgeirsson
E.R. Caianiello
V. Roglić
St. Fallieros
A. Gamba
R. Haag
Z. Janković
G. Lüders

Functions

Sen. Phys.

Dir. TH

Phys. TH (fellow

and later on member

of personnel

$1954(?))$

Secr. TH

Phys. TH fellow

(1.11.1953:

Engineer PS Group)

G.O.J von Gierke

B. d'Espagnat

K. Adler

A. Bohr

C. Møller

B.R. Mottelson

S. Rozental

E. Jacobsen

N.M. Hugenholtz

E. Eriksen

P.O. Fröman

D. Harting

G. Fidecaro

G.R. Bishop
Phys. TH fellow

Phys. TH

Phys. TH

Sen. Phys. TH

Sen. Phys. Dir. TH

(Copenhagen)

Sen. Phys. TH

Sen. Phys. Dep.-Dir. TH

(Copenhagen)

Secr. TH Copenhagen

Phys. TH

Phys. TH

Proposed by N. Bohr

in Oct. 1953 to be engaged

in Sept. 1954. Decision

postponed by Council, its session Oct. 1953. Was never engaged: accepted research fellowship at Oxford (Ref.

Weekly Report No 59, Jan. 54)
Nationality Date

DK

5.1952

Sw

1952

DK

F

UK

Iceland

I

Y

Gr

I

D

Y

D

$\begin{array}{lc}\text { D } & 1.3 .1953 \\ \text { F } & \text { Oct. } 1953 \\ \text { CH } & 1.9 .1954 \\ \text { DK } & 1.8 .1954 \\ \text { DK } & 1.9 .1954 \\ & \\ \text { USA } & 1.9 .1954 \\ \text { DK } & 1.9 .1954 \\ & \\ \text { DK } & 1954 \\ \text { NL } & 4.1954 \\ \text { Norway } & 1.1954 \\ \text { Sw } & 1.1954 \\ \text { NL } & 1.1954 \\ \text { I } & 9.1954 \\ \text { UK } & \end{array}$

UK
1952

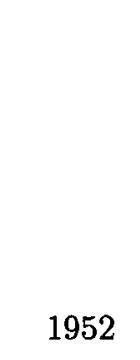

1.3.1953

8.1954

9.1954

954

1954

1954

1.1954 
I assume that all of them were paid by CERN but I am not sure that at the beginning there was any clear distinction between visitors paid by CERN and those paid by the Institute. However, soon things became more formal. An agreement was signed between the CERN Council and the Danish Government specifying the privileges and immunities of CERN employees in Copenhagen. Furthermore, the fellows were appointed by the Council following a proposal by Bohr. I have not found any case in which the Council acted contrary to Bohr's proposal, either by refusing to hire somebody or by imposing another choice, although occasionally they postponed a decision, often for financial reasons. In fact, in these early years, the Theory Group represented the only scientific activity of CERN and it was only normal that the Council followed closely its performance. In the minutes of the first sessions we find many references to discussions about the work of the Group, contrary to what happens today where the activity of the Theory Division, being a higher order correction to the CERN budget, is rarely discussed by the Council. As an example, in this year's document, presented by the Director General to the Scientific Policy Committee and the Finance Committee ('The Scientific activities of CERN and budget estimates for the years 1993-1996', CERN/SPC/646/Rev., CERN/FC/3543, 19 May 1992) the chapter concerning the Theory Division covers less than two pages out of a total of 84 pages. In these days everybody seemed concerned about attracting the most promising young theorists to work in Copenhagen. In a letter to R.W. Penney dated July 20, 1954, S. Rozental, then deputy-Director of the group, noted: '... In fact, I doubt whether we shall get the right people by applications; probably we shall have to try to find them ourselves .... The principle of roughly one fellow per member state seems to have been agreed upon already at that time. Visitors from non-member states were also accasionally admitted. In June 1956, the Ford Foundation offered CERN a $\$ 400000$ grant for a period of five years which was used to finance such visits. As years passed the group increased in size and, in September 1954, we find twenty-four theorists and two secretaries working in Copenhagen. Also from this date, C. Møller succeeded to Niels Bohr as head of the group. There was also a slight dispersion. A few theorists were dispatched to Liverpool, Glasgow or Uppsala. These centers opened their experimental facilities to European collaboration.

I believe that in Bohr's mind the Copenhagen arrangement was meant to be a long term one, but other delegates thought otherwise. For example, the British delegation submitted a note to the Council meeting of June 20, 1953 in Rome in which we read:

The United Kingdom point of view on this question is that a theoretical physics centre should be maintained at Copenhagen, as part of the European Organization, for a period of five years, the position then to be reviewed. The centre should not, in this period, build a permanent institute, but should if possible use existing premises. The period of five years is suggested to secure reasonable staffing stability.

It would be desirable to have some estimate of the cost to the European Organization unless details are already available. It is observed that according to $C E R N / G E N / 5$, the present budget estimates allow for an average total of about 
900000 Swiss Francs per annum to be spent on theoretical work and other forms of cooperation.

The French delegation responded with a similar note in which the proposed period was reduced to three years.

A theoretical study Group should be at work at Geneva when the construction of the Synchro-cyclotron is achieved, i.e. following the present evaluation, at the latest 4 years after the entry into force of the Convention. It will be therefore desirable to establish this Group during the year preceding the putting into operation of this accelerator. Consequently, the contracts to be eventually concluded with theoricians in Copenhagen, should be limited to a period of 3 years.

The amount of S.F. 900000 mentioned in chapter D of the programme proposed by the Council at its fifth session, as shown by the table on page 11 of document $C E R N / G E N / 5$, must be divided between the activities of the Copenhagen Institute and the cost of other forms of cooperation. Furthermore, the sums needed for the establishment and the gradual development of a theoretical Group within the Geneva Laboratory, ought to be reserved at an appropriate time.

It is also amusing to note that the cost of the Theory group represented at that time the main item in the CERN budget! As things finally worked out, the agreement with Copenhagen lasted five years. With an official letter dated January 16, 1957, C.J. Bakker, then Director General, informed the Danish Government of the Council's decision to terminate the agreement with Copenhagen on October 1st, 1957. As of this date, the Theory Division joined the laboratory in Geneva.

What was the scientific activity of the group during the Copenhagen period? We have ample information on this question because Rozental was submitting detailed scientific reports to the Council every three months with full lists of publications, seminars, colloquia, etc. The first thing we notice is that, to a large extent, the Group acted as a training center for young theorists. This is not surprising. In postwar years, Physics education in most European Universities left a lot to be desired. Chairs were few and often held by old Professors who had not kept up with the latest scientific developments. Organized graduate studies were almost absent. It was not exceptional to see Research Centers playing the role of Graduate Schools. For example, in France, A. Messiah taught the course, which gave rise to his textbook on Quantum Mechanics, not in a university but in Saclay. Generations of French Physicists had to learn Modern Physics in the Summer School of Les Houches. In the Rozental reports we repeatedly see courses on rather elementary subjects, such as the classic 'Angular Momentum in Quantum Mechanics', taught in the Center. Presumably, for many young visitors, their position in Copenhagen was that of a graduate student rather than of a postdoctoral fellow.

Nevertheless, the group was active in research. A substantial part was devoted to theoretical Nuclear Physics, a choice which reflects both the scientific interests of many senior physicists in Copenhagen, as well as the only experimental activity in Europe outside cosmic ray physics. Strictly speaking, it is in this field that the most significant scientific work was done. A. Bohr and B.R. Mottelson had just completed their work on nuclear levels which, later, gave them the Nobel prize. In 
the early fifties, the subject was in full expansion with many experimental groups producing new results. A large part of this theory was elaborated and applied to particular nuclei by the Copenhagen Group. However, I do not know whether we can consider it as a CERN activity in view of the fact that the principal investigators belonged to the Copenhagen permanent staff and the subject was essentially dropped once the group moved to Geneva.

Quantum Field Theory and Elementary Particle Physics, the subjects that would become the main scientific activity of the CERN Theory Division, were also present during the Copenhagen years. Partly in order to appreciate correctly the significance of their contributions and partly for future reference, we shall briefly present the international scene during the same period.

The fifties must have been extremely exciting years for Elementary Particle Physics. It was during this period that the subject became an independent, fully grown discipline. This was mainly due to the systematic use of accelerators which marked the separation from Cosmic Ray Research and set the era of Big Science.

The decade witnessed the growth of pion physics, the study of the first pionnucleon resonances and the discovery of antinucleons. The first heavy flavors were introduced and understanding their deep significance still lays heavily upon us. The triumph of renormalization theory created enthusiasm followed by depression. Parity was found to be violated and the V-A theory was established. The fundamental role of symmetry was recognized and the first non-Abelian gauge theories were constructed. A detailed account of all these wonderful adventures goes far beyond the scope of this essay, so we shall only say a few words about them. Since this chapter is about Theory, I shall follow the development of theoretical ideas.

The so-called 'modern era' of Quantum Field Theory has a precise starting date: June 2, 1947, the date of the Shelter Island Conference. The most important contribution that was presented at this Conference was not a theoretical breakthrough but an experimental result: Willis Lamb, of Columbia University, reported the measurement of an energy difference of about $1000 \mathrm{MHz}$ between the $2 S_{1 / 2}$ and $2 P_{1 / 2}$ levels of the hydrogen atom, in contradiction with the Dirac theory. The 'Lamb shift' shows the real nature of the effects of vacuum polarization. Its importance is not that it was unexpected. As Steven Weinberg puts it: '... It was not so much that it forced us to change our physical theories, as that it forced us to take them seriously.' In the months which followed, Richard Feynman and Julian Schwinger set the foundations of the theory of renormalization, a welldefined prescription to compute the higher order terms in the perturbation series of quantum electrodynamics. As it turned out, similar ideas had been developed independently in Japan by Sin-Itiro Tomonaga, who was the first to give the complete computation of the Lamb shift. The entire program was formally described by Freeman Dyson in 1949. Quantum Electrodynamics, the theory of interacting photons and electrons, supplemented with the program of renormalization, is one of the most successful theories in physics. Its agreement with experiment is spectacular. But it was also the first successful Quantum Field Theory, the quantum mechanics of a relativistic system with an infinite number of degrees of freedom. Although the essential ideas were already developed in the late forties, a large 
amount of work was still necessary for the theory to be completed. The more technical part of the renormalization program was elaborated by Bogoliubov and Parasiuk in the Soviet Union around 1955 and by Klaus Hepp in Zürich in the sixties. The application of the program to meson theories was done by Matthews and Abdus Salam in Great Britain in 1949-1951. Some of the ground work, including, in particular, the correct form of the propagator in Quantum Field Theory, was laid down by E. Stückelberg at the University of Geneva, who must also be credited, together with A. Peterman, with the introduction of the concept of the renormalization group in 1954 .

The contribution of the Copenhagen group to this effort was rather modest. I have singled out an article by A. Peterman who was a fellow from Switzerland. In 1957, he published one of the first two-loop calculations of the electron anomalous magnetic moment [1] (the classical one-loop calculation was done by Schwinger). Although with modern techniques, such computation sounds rather simple (for comparison, the four-loop calculation was completed a few years ago), it represents a milestone in our understanding of Quantum Electrodynamics. First of all, it required a mastery of the intricacies of the renormalization program which few theorists possessed at the time and, second, the actual calculations, both analytic and numerical, were quite involved. The result confirmed the agreement with experiment.

As already said, the success of quantum electrodynamics is that of renormalized perturbation theory. It is the same approach which, to our great surprise, led to the revolution of gauge theories and the construction of the Standard Model. Even today, we do not understand why this approach works so well, neither do we know whether realistic quantum field theories exist outside perturbation. It was precisely during the fifties that the axiomatic formulation was initiated. In this line of thought the Copenhagen contribution is very significant. In 1955, Rudolf Haag published a classic paper [2], the first in a series, in which he set the mathematical foundations of scattering in the framework of quantum field theory. He showed the impossibility of accommodating, in the same Hilbert space, the bare and the renormalized vacuum states, thus showing that the socalled 'interaction representation' was a very ill-defined concept. For the first time, he used sophisticated mathematical techniques borrowed from the theory of topological vector spaces at a time when topology was terra incognita to most theoretical physicists.

The second 'exact theorem', concerning quantum field theory, that was published by the Copenhagen group is due to Gerhart Lüders. Starting from the pioneering works of Eugene Wigner, theorists had studied the invariance properties of quantum field theories under the discrete transformations of space inversion or parity $(\mathrm{P})$, time reversal $(\mathrm{T})$ and particle-anti-particle conjugation $(\mathrm{C})$. These transformations present a special interest in the framework of a quantum theory as compared to that of the classical case. A profound theorem was proven, called the 'CPT theorem', which states that, under very general assumptions such as relativistic invariance, locality and positivity, the product of these three operations is an invariance of the world. It implies, in particular, the exact equality of masses and life-times between particles and anti-particles. There exist several 
proofs of this theorem and one of them is due to Lüders who was in Copenhagen in 1954 [3]. The problem was suggested to him by Bruno Zumino and it is amusing to notice that the title of the paper is 'The equivalence of invariance under time reversal and under particle-anti-particle conjugation for relativistic field theories'. Invariance under parity was taken for granted! It is not mentioned as a specific assumption in the abstract and it is only at the end of the second page that we learn, without any particular emphasis, that for the validity of the proof, the theory must be invariant under space reflexions. Apparently, parity violating theories were not considered worth studying. And this happened in 1954, just two years before the earthquake.

The enthusiasm raised by the successes of quantum electrodynamics did not last long. It was natural for theorists to try to apply the same techniques of renormalized perturbation theory to the other elementary particle interactions, namely the strong and the weak ones. In fact, a large amount of work was done concerning meson-nucleon theories which were supposed to describe nuclear forces. The results were disappointing. At the formal level the renormalization program was shown to be applicable to a particular form of the coupling (the so-called pseudo-scalar theory) but the results were useless for practical computations. The reason is a profound difference in the strength of strong interactions as compared to the electromagnetic ones. The perturbation expansion is a formal power series in a dimensionless parameter, the renormalized coupling constant. Its numerical value is determined by experiment. For quantum electrodynamics, this value turns out to be roughly equal to $1 / 137$, the celebrated fine structure constant. The smallness of this number is responsible for the success of the theory since higher order terms soon become negligible and a good estimate of the result can be obtained by keeping only the first few terms. The corresponding number for pion-nucleon interactions is instead of the order of 10 and the very idea of a power series expansion becomes meaningless. This is, of course, the reason why strong interactions are strong! The dynamics is often dominated by resonance production, a phenomenon completely outside the scope of perturbation theory. For a few years this caused a considerable confusion among theorists which was soon resolved by abandoning field theory techniques in strong interactions. Few theoretical contributions to this subject can be considered as being significant and I have not found any of these coming from Copenhagen.

What about the weak interactions? Since they were supposed to be much weaker than the electromagnetic ones, this objection did not apply. However, a new theoretical problem appeared there: since 1934, Enrico Fermi had proposed a remarkably simple and elegant field theory model which seemed to describe perfectly well all known phenomena of weak interactions. It became known as 'the four-fermion theory'. The trouble is that, as was immediately realized, the renormalization programme did not apply. One could not resolve all the ambiguities, to any given order of perturbation theory, by redefining only a finite number of constants. The theory was in fact lacking any predictive power.

This double failure soon tarnished the glory of quantum electrodynamics. The general disappointment was such that renormalization theory became an essentially esoteric topic in particle physics. When I was studying in Graduate School, 
in the early sixties, the subject was not even taught in most universities. New approaches were developed, all of which were initiated in the United States. In fact, as years passed, Europe, if anything, seemed to be losing ground!

For strong interactions these new approaches involved two concepts: internal symmetries on the one hand and analytic properties of the S-matrix on the other. It is amusing to notice that Werner Heisenberg can be considered as the father of both, because he was the first to introduce a sort of isospin symmetry for nuclear forces in the thirties as well as to use S-matrix ideas in a remarkable paper written during the war. However, it is fair to say that, in their modern form, they were both developed in the United States.

I believe that the milestone of the symmetry approach is a little-known work of Fermi. In 1952, he used a simple isospin symmetry argument, in order to deduce the $I=\frac{3}{2}$ dominance in $140 \mathrm{MeV}$ pion-nucleon scattering. He had been studying pion interactions at the $450 \mathrm{MeV}$ Chicago synchro-cyclotron. The cross sections for $\pi^{+} p \rightarrow \pi^{+} p\left(\sigma^{++}\right), \pi^{-} p \rightarrow \pi^{\circ} n\left(\sigma^{-o}\right)$ and $\pi^{-} p \rightarrow \pi^{-} p\left(\sigma^{--}\right)$at their highest pion energies $(140 \mathrm{MeV})$ were measured to obey approximately $\sigma^{++}$: $\sigma^{-\circ}: \sigma^{--}=9: 2: 1$ which follows from isospin only if $I=\frac{3}{2}$ dominates. In the same year, K.M. Watson proved that the average numbers of pions produced in nucleon-nucleon collisions satisfy the relation $\bar{n}^{+}+\bar{n}^{-}=2 \bar{n}^{\circ}$. Simple ratios of Clebsh-Gordan coefficients! It was the first time that results of this kind were obtained in particle physics from symmetry principles alone, independently of any detailed dynamical model! From this time Symmetry became the name of the game! It entered the world of particle physics and proved to be the most profound and most powerful concept. At the beginning, its role appeared to be limited because most symmetries were assumed to be global. As such they had little influence on the underlying dynamics. They could only provide relations among masses or amplitudes. It took many years for physicists to realize that in the framework of gauge theories, it is symmetry which determines the dynamics. But more about this later.

In the symmetry approach I want to mention one contribution coming from Copenhagen. In 1953, Louis Michel, a French theorist, already well known from his analysis of the electron spectrum in muon decay, introduced the ingenious concept of what became later known as G-parity, a combination of charge conjugation and a $180^{\circ}$ rotation in isospin space [4]. This simple idea had a considerable impact on pion physics because now, all pions, neutral as well as charged, were eigenstates of G-parity. Furthermore, as it turned out, it was straightforward to generalize this concept to higher symmetries.

The analyticity approach was from the start more ambitious. I believe that its origin should be traced back to the realization that the analyticity properties necessary to derive the Kramers-Kronig dispersion relation for the scattering of light by matter, can be obtained from causality without the use of detailed dynamical assumptions. I do not know who is the father of this important observation which may go back as far as the twenties. But in strong interactions, the first dispersion relations were written down by $M$. Goldberger in 1955 for the pion-nucleon forward scattering amplitude. The importance of this work can hardly be overestimated. Few papers in particle physics have had comparable in- 
fluence and in a few years this approach hac become the main school of thought in strong interactions. Its domination was such that it reached occasionally almost religious dimensions. Europe in general and the CERN Theory Division in particular, played an important role in its development, but not during the Copenhagen years. So I shall leave this story here and pick it up again later.

Maybe the most spectacular progress that was made in theoretical particle physics during the fifties was in the physics of strange particles and the properties of weak interactions. It was one of these fortunate circumstances in which cooperation among theorists and experimentalists proved to be most fruitful. Unexpected experimental results gave rise to novel theoretical ideas which in turn suggested new experimental research. Much to my regret, I shall not tell this wonderful story in any detail because I must admit that the role of Europe in it was not very brilliant.

The first evidence of 'heavy flavors' goes back to 1936 with the discovery of the muon. In retrospect it marked a new era in physics. It was the first 'elementary particle' whose role in the structure of the world was, and still is, mysterious. Just after the war, the first hadrons with 'strange' properties appeared also in cosmic rays. Quite surprisingly, their behaviour seemed to depend on whether one looked at their production or their decays. They were copiously produced, which meant that they had strong interactions, but they were long-lived with life-times characteristic of weak interactions. The puzzle was eventually solved between 1953 and 1956 with the introduction by Murray Gell-Mann and K. Nishijima of a new quantum number, called 'strangeness' which was assumed to be conserved by the strong and electromagnetic interactions but violated by the weak ones. The Physics of strange particles soon became an invaluable source of information for experimentalists and a choice field for theorists. The highlights of this story are the following: (i) Murray Gell-Mann and Abraham Pais noticed that the two neutral $K$-mesons $K^{\circ}$ and $\overline{K^{\circ}}$ with well-defined strangeness quantum numbers, are not eigenstates of the total hamiltonian since weak interactions do not conserve strangeness and allow for virtual $K^{\circ} \Longleftrightarrow \overline{K^{\circ}}$ transitions as well as $K$ decays. The states with well-defined life-times are instead $K_{\mathrm{S}}^{\circ}$ and $K_{\mathrm{L}}^{\circ}$ which are two linear superpositions of $K^{\circ}$ and $\overline{K^{\circ}}$. (ii) A. Pais and O. Piccioni predicted the phenomenon of regeneration, the fact that a $K_{\mathrm{L}}^{\circ}$ beam passing through a slice of matter regenerates a $K_{\mathrm{S}}^{\circ}$ component. Beautiful examples of simple Quantum Mechanics! (iii) On the experimental side, a new puzzle soon came up: it is known as the ' $\tau-\theta$ puzzle' from the names of two decay modes of what seemed to be one and the same particle. The $\theta$ decay was a two-pion mode of a charged, strange meson. The $\tau$-mode was a three pion one. All studies (mass, life-time, scattering properties) indicated that the same $K$-meson was responsible for both. Using a novel method to analyze three-body decays (the 'Dalitz-plot') one could show that the $K$-meson had zero spin. The three pion mode implied that it had negative parity. But then parity conservation forbids the two-pion mode. That was the puzzle! (iv) All attempts to resolve the puzzle by 'conventional' means failed. The answer turned out to be simple, unbelievable and profound. In 1956 two Chinese born American theorists, T.D. Lee and C.N. Yang went through the literature on $\beta$-decay. To their astonishment, they found that, up to that moment, 
there was absolutely no evidence that parity was conserved in weak interactions. They made the bold proposal that it was not! Next year, it was proven beyond any doubt that they were right! Specifically designed experiments, involving polarized initial states, showed that the violation was maximal in $\beta$ as well as $\mu$ decays. A most astonishing result! A physical state is not equivalent to its mirror image! (v) The last point I want to mention here is the search for an understanding of the nature of weak interactions. It has been one of the most exciting and most rewarding enterprises in the history of elementary particle physics. The road was long and circuitous and many a time it seemed that it was leading to a dead end. Out of the struggle for such an understanding grew many ideas, the importance of which transcends the domain of weak interactions and covers most of modern physics. A detailed history of this adventure has not yet been written. When it is written, it will appear as a play in several acts. I shall only give here the titles. The first act culminates in 1934 with Fermi's four-fermion theory, a most extraordinary paper, written just after the discovery of the neutron and the conjecture about the neutrino. The second is called 'twenty years later' and the main actors are experimentalists. It is about the struggle to determine the form of the interaction. It ends around 1957 with the establishment of $\mathrm{V}-\mathrm{A}$. Some of the experiments necessary to that end will remain as monuments in the history of physics (example: Goldhaber's measurement of neutrino's helicity). The third act is a very short one: it has a prologue by S.S. Gershtein and Y.B. Zel'dovich in the Soviet Union and a main part by M. Gell-Mann and R.P. Feynman in the United States. It solves exactly one half of the problem of determining the weak interaction hamiltonian. The punch line is C.V.C. (Conserved Vector Current) and it identifies the vector part of the strangeness conserving weak current with the isospin current of strong interactions.

This is obviously not the end of the play but it is time for an intermezzo. We are in 1957 , i.e. time to move to Geneva. Until now, Copenhagen has not been part of the scenery in this weak interaction play.

Let me attempt to summarize: within the five years the CERN Theory Group stayed in Copenhagen, I have singled out four papers in field theory and particle physics: Peterman's calculation of $g-2$, Haag's rigorous formulation of scattering theory, Lüders' CPT theorem and Michel's G-parity. To those who may think that four papers in five years sound like a poor harvest, let me remind that their contents can be found in today's textbooks. A very severe selection criterion! I do not know how many papers from our present prolific literature will be parts of the textbooks within twenty-five to thirty years from now. My judgement is that, as far as the level of scientific output is concerned, the Center was a success. My criticism is that, only in nuclear physics, did it succeed in creating a School. With the obvious danger of exaggeration, I would say that all the important work in particle physics was done by individuals who happened to visit Copenhagen. None stayed long enough to attract other young fellows and create a group. I suspect that this is also due to the fact that the nuclear physics attractor was locally too strong, in any case the situation changed when the group moved to Geneva. 


\section{Moving to Geneva}

In 1954, Felix Bloch was appointed as the first Director General of CERN. He was supposed to go to Geneva because construction was planned to start soon. He was not very enthusiastic with the idea of being alone with only construction engineers to talk to, so he asked the Council permission to bring two theorists with him. He first invited Anatole Abragam from France. Abragam was not a high energy physicist. Like Felix Bloch, he was a very well-known specialist of nuclear magnetism. He was head of a group at Saclay and promised to come to Geneva only part-time. In fact he never stayed for long and, in any case, Bloch resigned his post and returned to Stanford the year after. As a result, Abragam had even less motivation to visit CERN and he rarely did. Let me only mention that his work has influenced the experiments in the laboratory in later times, because he developed novel methods to build polarized targets. But this chapter is about theory. The first theorist to join CERN in Geneva was Bernard d'Espagnat. He had been in Copenhagen as fellow and was highly recommended to Bloch by Møller. With a letter dated July 10, 1954, Bloch offered him the position starting October of the same year. A few months later, on January 1955, d'Espagnat was joined by a second theorist, Jacques Prentki. D'Espagnat and Prentki both came from Leprince-Ringuet's Laboratory at Ecole Polytechnique in Paris. They had been working in the physics of strange particles and in the theory of weak interactions, the 'hottest' subjects of the time, and they continued their collaboration in Geneva. In fact the work they did together during the next years is probably CERN's most significant theoretical contribution of the early times. I shall present here two main ideas: the first is an attempt to find a geometrical meaning to the conservation of strangeness in strong interactions and the second is a search for the symmetries of weak interactions.

Strong interactions were known to conserve electric charge and baryon number. Electric charge conservation was generalized quite early to a full invariance under three-dimensional rotations in isospin space, a non-Abelian symmetry. Isospin, electric charge and baryon number were not, however, independent symmetries, they were related by $Q=I_{3}+B / 2$, where $Q, I_{3}$ and $B$ denote the electric charge, the third component of isospin and the baryon number, respectively. Although I do not know who first wrote down this simple relation explicitly, it was generally assumed to be correct. The electric charge of a particle determined the isospin multiplet to which it belonged. This connection was broken with the introduction of strangeness. Already in his 1953 paper, Gell-Mann proposed to assign half-integer isospin to $K$-mesons and integer isospin to $\Sigma$ and $\Lambda$ hyperons, although they were all integrally charged. The relation was eventually modified to $Q=I_{3}+\frac{S}{2}+\frac{B}{2}$ where $S$ denotes strangeness, a new quantum number. Thus the conservation of strangeness can be seen as a decoupling between the conservations of the other three quantities which now become independent. The quest for higher internal symmetries dates from this observation. It was one of the major theoretical problems of this period and d'Espagnat and Prentki made some very important contributions. (The problem was eventually solved by M. Gell-Mann and Y. Ne'eman in the early sixties with the introduction of unitary symme- 
try). Two directions were explored: in a 'conservative' approach, d'Espagnat and Prentki attempted to enlarge the symmetries of strong interactions by considering reflexions, together with rotations, in the three dimensional isospin space [5]. The idea was based on the ingenious observation that the phase transformations responsible for electric charge conservation were in fact equivalent to reflexions in isospin space, provided one used spinors of the second kind to describe $K$-mesons and cascade baryons. They wrote down the most general interaction Lagrangian invariant under isospin rotations and reflexions and showed that it automatically conserved strangeness. They even used their formalism in order to predict the possible existence of an isoscalar $0^{-}$meson several years before the discovery of $\eta$ and $\eta^{\prime}$. As far as I know, they were the first to make such a prediction.

This 'minimal' scheme was not a very powerful one. In particular, it made no prediction concerning the masses of particles belonging to different isospin multiplets. In 1957, d'Espagnat and Prentki, in collaboration with A. Salam, considered a more 'radical' approach in which the symmetry group of the strong interactions was increased from $S U(2)$ to $O(4)$ [6] (I believe that the idea of an internal symmetry higher than isospin is due to A. Pais, who, already in 1954, proposed a first version of $O(4))$. The algebra of $O(4)$ is that of $S U(2) \times S U(2)$ i.e. that of two isospin groups. Therefore, one has two 'third isospin components' to connect with electric charge and strangeness. Several versions of $O(4)$ have been published by many authors differing in the representation assignment of the various particles and/or the compact or non-compact form of the group. However, as far as I know, d'Espagnat and Prentki, following their work with Salam, were the first to address in detail the question of the symmetries of weak interactions. This brings me to their second major contribution which is beautifully summarized in their 1958 Nuclear Physics paper [7]. 'A tentative general scheme for weak interactions'. This paper contains a comprehensive picture of the symmetries of all interactions. For the first time the whole hierarchy of symmetries and interactions is clearly presented. Four levels are considered:

1) The very strong interactions are invariant under $O(4)$.

(2) The medium strong ones break $O(4)$ and leave one $S U(2)$ (isospin) and the third component of the other (strangeness) invariant.

(3) The electromagnetic interactions conserve only the third components of the two $S U(2)$ 's (electric charge and strangeness).

(4) Finally, the weak interactions were supposed to be invariant under a different $S U(2)$ subgroup of $O(4)$.

Thus strangeness violation was the result of a mismatch between the medium strong and the weak interactions. Let me add here that the idea of introducing medium strong interactions was already known, but in its early versions it was supposed to describe the interactions of $K$-mesons as opposed to those of pions which were the very strong ones. The correct scheme, as we know it today, appeared for the first time in d'Espagnat and Prentki's paper. Incidentally, I never quite understood why these authors, after having written this extremely lucid and beautiful paper, failed to discover the Cabibbo theory immediately 
after the introduction of $S U(3)$. In fact, they almost did so! Let me anticipate in time and describe a 1962 paper [8], published in Il Nuovo Cimento, in which they reconsider their theory in the language of $S U(3)$. This is the most amazing part of the story! In section 3 of this paper one finds explicitly the correct form of the Cabibbo current with an arbitrary angle called $\alpha$. Then they proceed to show that in a current $\mathrm{x}$ current theory the two empirical selection rules $|\Delta S| \leq 1$ and $|\Delta I|<\frac{3}{2}$ for non-leptonic processes are related. And they stop there! They do not mention that this form of the current allows for an unambiguous definition of the concept of universality, a very important element of the Cabibbo theory, and that one can actually determine the weak angle by looking at semi-leptonic decays. In their paper leptons are only mentioned in the last paragraph. It seems that they were misled by an erroneous experiment claiming a large $\Delta S=-\Delta Q$ admixture in strangeness changing semi-leptonic processes.

Since I went that far, let me also give the end of the story. The following year, a short paper appeared, again as a CERN preprint [9]. The author was a young visitor from Italy, Nicola Cabibbo. He took over the idea of a current which forms an angle with respect to medium strong interactions, but he carried it on to its logical conclusion. This form allows for the only consistent definition of universality. The concept of universality had been introduced several years before and it was often expressed by the vague assumption that all possible weak processes had the same strength. Cabibbo noticed that this assumption did not make sense. Using modern quark language, his remark can be translated into the statement that, with one quark of charge $\frac{2}{3}$ and two quarks of charge $-\frac{1}{3}$, one could always construct one hadronic current which is coupled to leptons and one which is not. He chose to define universality by the assumption that the coupled current involves the same coupling constant as the purely leptonic processes. He then proceeded to compare strangeness conserving and strangeness changing semi-leptonic decays and he deduced the value of what became known as the 'Cabibbo angle'. I do not know whether he was unaware of the wrong experimental result or whether he showed the good physical judgement to ignore it, but he clearly understood that the semi-leptonic processes were the important ones. There are very few articles in the scientific literature in which one does not feel the need to change a single word and Cabibbo's is definitely one of them. With this work he established himself as one of the leading theorists in the domain of weak interactions.

After this short excursion, let me come back to history. In 1957, the relative isolation of d'Espagnat and Prentki ended. CERN's headquarters were now officially in Geneva, and all its employees came there. They included the theorists from Copenhagen as well as new appointments. The group expanded rapidly, left the provisional barracks it occupied near the airport and moved to more permanent premises in Meyrin. The evolution of the Division is shown in the following two Tables. The first gives the composition in June 1959 under the direction of Markus Fierz. The second contains all physicists who have had permanent appointments. 
Staff, TH Division as on June 121959

$\begin{array}{ll}\text { M. FIERZ } & \text { Director } \\ \text { B. d'ESPAGNAT } & \text { Senior } \\ \text { S. FUBINI } & \begin{array}{l}\text { Staff } \\ \text { J. PRENTKI }\end{array} \\ & \text { Members } \\ \text { D. AMATI } & \text { A. MARTIN } \\ \text { R. ASCOLI } & \text { Ph. MEYER } \\ \text { J.E. BOWCOCK } & \text { G. MOLIERE } \\ \text { F. CERULUS } & \text { A. PETERMANN } \\ \text { D. FELDMAN } & \text { MrS. SALZMAN } \\ \text { V. GLASER } & \text { G. SALZMAN } \\ \text { C. FRONSDAL } & \text { R.F. SAWYER } \\ \text { D. GEFFEN } & \text { D. SPEISER } \\ \text { R. HAGEDORN } & \text { R. SHERR } \\ \text { E.M. HENLEY } & \text { F.L. SCARF } \\ \text { B. JAKSIC } & \text { W. THIRRING } \\ \text { R. JOHNSTON } & \text { H.A. TOLHOEK } \\ \text { J.M. JAUCH } & \text { B. VITALE } \\ \text { T. KANELLOPOULOS } & \text { J.D. WALECKA } \\ \text { W. KLEIN } & \text { J. WESS } \\ \text { S. KOHLER } & \text { K. WILDERMUTH } \\ \text { D. LURIE } & \text { Y. YAMAGUCHI }\end{array}$

NAME

ARRIVAL NAT

$\begin{array}{llcl}\text { HAGEDORN } & \text { Rolf } & 1 / 04 / 54 & \text { DE } \\ \text { PRENTKI } & \text { Jacques } & 1 / 01 / 55 & \text { FR } \\ \text { PETERMANN } & \text { André } & 1 / 09 / 55 & \text { CH } \\ \text { GLASER } & \text { Vladimir } & 1 / 10 / 57 & \text { IT } \\ \text { AMATI } & \text { Daniele } & 1 / 02 / 59 & \text { IT } \\ \text { MARTIN } & \text { André } & 1 / 01 / 60 & \text { FR } \\ \text { BELL } & \text { John } & 11 / 01 / 60 & \text { GB } \\ \text { VAN HOVE } & \text { Léon } & 4 / 09 / 60 & \text { BE } \\ \text { ERICSON } & \text { Torleif } & 5 / 09 / 60 & \text { SE } \\ \text { JACOB } & \text { Maurice } & 1 / 06 / 67 & \text { FR } \\ \text { ZUMINO } & \text { Bruno } & 29 / 05 / 69 & \text { US } \\ \text { FUBINI } & \text { Sergio } & 1 / 06 / 73 & \text { IT } \\ \text { ELLIS } & \text { John } & 1 / 09 / 74 & \text { GB } \\ \text { VENEZIANO } & \text { Gabriele } & 1 / 07 / 77 & \text { IT } \\ \text { DE RUJULA } & \text { Alvaro } & 6 / 09 / 77 & \text { ES } \\ \text { FERRARA } & \text { Sergio } & 1 / 03 / 81 & \text { IT } \\ \text { ALVAREZ-GAUME } & \text { Luis } & 23 / 06 / 86 & \text { ES } \\ \text { ALTARELLI } & \text { Guido } & 1 / 03 / 87 & \text { IT }\end{array}$


Some remarks: the arrival dates shown are supposed to represent their first affiliation with CERN. Indefinite contracts were not granted until later. (CERN's policy for indefinite appointments was not specific to the Theory Group and has been reviewed elsewhere). I do not know exactly what the date June 1st, 1973 represents for Sergio Fubini, but his connection with CERN is much older. He joined the Theory Group in November 1957 and stayed as a full-time member until February 1960. Between 1960 and 1964, he was partly at CERN and partly in Padua, he left for Italy and the United States in 1964 and came back to CERN in 1971. Rolf Hagedorn's first appointment at CERN was in the PS Division where he was in charge of computing the multiparticle cross-sections and distributions. Since this work gave rise to some important concepts in high energy physics, I shall have the opportunity to mention it again later. His indefinite appointment in the Theory Division dates from January 1961. Bernard d'Espagnat is not mentioned among the permanent members. With a letter dated May 25, 1959 he resigned his position at CERN in order to accept a 'Maîtrise de conférences' at the newly founded campus of the University of Paris at Orsay. I consider this move as unfortunate. First, because it broke a very successful and fruitful collaboration. Although d'Espagnat continued to visit CERN, his collaboration with Prentki slowly faded away. Their last joint paper that I remember dates from 1963. Neither of them singly did subsequently as interesting and important work, as the one they had done together. Secondly, because they represented a line of research, that of weak interactions and symmetries, which came out considerably weakened. Leaving aside the question of relative personalities, a simple counting shows that the majority of new appointments at CERN was not in this direction.

One of the most influential members of the Theory Division does not appear in the tables. It is Tatiana Fabergé, who joined the Group as Secretary in March 1957. Few contributed to the shaping of the Division as much as she did, and, in fact, her influence has extended far beyond the CERN boundaries to the international community of theoretical high energy physics. She organized all the administrative work, which turned out to be absolutely essential for the rapidly expanding Division, she introduced the preprint numbering system which is still in use today and which has been since copied by many other institutions, but, above all, she succeeded in creating around the Theory Division Secretariat an atmosphere of friendship cherished by a whole generation of physicists.

The present preprint numbering system started in 1958. The paper with the number Th01 has a date of Oct. 30, 1958 and contains some lecture notes to P.S. on strange particle physics with an introduction by J.M. Jauch. The expansion of the Division can be inferred by following the Th preprint numbers. By the end of 1959, we are still in Th12, in 1960 we reach Th79, in 1961, Th155 and at present the number approaches Th7000.

An important turning point in the history of the Division is the appointment of its first Director. Until 1960, it has functioned under interim directors, first Niels Bohr and C. Møller in Copenhagen and then Bruno Ferretti and Markus Fierz in Geneva. None of these appointments was meant to be a long term one. The subject appears in the minutes of practically every session of 
the Scientific Policy Committee (S.P.C.) since 1956 as well as in those of many Council meetings. Many European and American physicists were consulted and were asked to nominate possible candidates. The position was offered to several leading theorists. We shall have an opportunity later to comment on the difficulties encountered in this process but I want to emphasize at this point that the long-term vacancy of the position was not the result of negligence or lack of coordination of the CERN authorities. The chairman of the SPC at the time was W. Heisenberg and one sees through the documents that he spared no effort to find a high level particle theorist who would be willing to take the job. After many unsuccessful attempts the offer was made to Léon Van Hove from Belgium. Van Hove was not a high energy physicist. Although quite young, he was already well-known, mainly for his work in Statistical Mechanics. His name is still associated with the thermodynamic limit in an interacting system. The problem is a very fundamental one because it raises the question of the very applicability of statistical mechanics to the description of phase transitions, given the fact that the partition function for a finite system is an analytic function of the parameters of the theory. In the forties it was not yet fully realized that discontinuities can emerge when one takes the limit of an infinite system. Whether and how this can be done was answered by Van Hove in a pioneering 1949 paper. Apparently, several members of the SPC were worried that his lack of expertise in CERN's field of research would prevent him from fulfilling the task assigned to the Director of the Theory Division, which consisted in conducting high level research, attracting new theorists, directing the research of the young fellows and taking an active part in the establishment of the laboratory's experimental programme. Van Hove was finally contacted in 1959 and came to CERN in September 1960. His appointment was initially for one year and became indefinite the year after. It is plausible that Heisenberg, or somebody else, transmitted to Van Hove the reservations that were formulated in the SPC on his account, in any case this appointment marked a turning point in the scientific orientation of the young Division leader. Not only did he stop working in statistical mechanics and embrace particle physics, but also his very style of work changed. From abstract and mathematical it became phenomenological following closely the experimental results. I shall describe in the next section some of his work on multiparticle dynamics which, however, is not at the same level as his earlier rigorous results in statistical mechanics. Let me only remark here that he exercised a great influence on the development of the Theory Division, both through his position as Director, as well as through his strong personality.

\section{CERN, the Center of Europe}

This section will cover the decade from 1960 to 1970. The first thing that should be explained is the title. To a large extent, it applies to the entire Laboratory, but, obviously, I shall only refer here to the Theory Division.

It is impossible to assign a precise date at which an institution reaches the age of maturity, but for the CERN Theory Division, this date can be placed around 1960. The nineteen fifties were the years of preparation and, to some extent, 
the years of uncertainty. CERN was the first example of international scientific cooperation and for many physicists its future was not guaranteed. Being a new venture it did not have the prestige of a University chair. If experimentalists were often attracted by the facilities the Laboratory could provide, many theorists preferred even a junior faculty position to a CERN staff membership. Under these conditions, it is no wonder that the Council had such great trouble in finding a Director for the Theory Group. Well-established theorists were not willing to give up their prestigious chairs.

This situation changed radically around 1960. Several reasons concurred to this. First, the experimental facilities were completed and the first results were obtained. Although few great discoveries were made, (I can only think of the decay mode $\pi \rightarrow e \nu$ which was found at the SC and was missed in the United States) the work of the Laboratory had gained international recognition. The Theory Division had expanded and developed a very active and very rich visitors' programme. This should be contrasted with what happened in most European Universities where visiting positions were extremely few if not totally unavailable. By the mid-sixties, CERN had most probably the largest theoretical high energy physics department in the world. The fellowships reserved to post-docs from the member states made Geneva the meeting place of the best young theorists of Europe. Practically all leading European and American physicists would visit CERN regularly. Especially during the summer months, all available desk space was used. There were even occasional angry letters by American theorists whose applications for unpaid visits were turned down. As a fellow theorist has put it, everybody who was somebody had to visit CERN. An invitation to give a seminar at CERN was considered as a great achievement. Furthermore, at least as far as theory was concerned, the entire enterprise was left to the young generation. I remember my first visit to CERN while I still was a graduate student. I was sitting in the back row of the seminar room and remarked that I could not see a single white hair. Coming from a country in which a University Professor was never below fifty, I was very impressed.

All this made the Theory Division a very exciting and stimulating place. Gone were the days when one '.. could not get the right people by applications'. A CERN staff membership was an offer one could not refuse. I know of many physicists who turned down a CERN offer in the fifties. I also know quite a few who did so in the seventies and eighties. There is hardly any in the sixties. Let me also emphasize that, contrary to a wide-spread belief, CERN salaries at that time were not significantly higher than those in the rest of Europe. When I moved to CERN as a post-doc in 1966, I received a very small raise as compared to my French beginner's salary. CERN salaries were driven up by the spectacular rise of the Swiss franc which only occurred in the very late sixties and the seventies. In 1966, however, the French and the Swiss francs were approximately equal.

As a result, during the sixties, CERN in general and the Theory Division in particular, were the undisputable scientific centers for high-energy physics in Europe. This domination was such that it gave rise occasionally to personal conflicts and tensions. Some theorists resented the fact that CERN had made no offer to them. This was accentuated by the very rapid initial expansion which 
created an essentially frozen situation during the sixties. I do not know whether a different policy, namely a more gradual expansion, would have been preferable. It would have certainly left room for a more equilibrated spectrum, both with regard to age and scientific interests, but on the other hand, an initial critical mass was necessary for the success of the enterprise. Quite apart from this question, one should also acknowledge the very essential role that CERN played as a training center for European theorists. If after 1970 it ceased to occupy the unique position it had during the sixties, this is, to some extent, the ransom of success.

So much for the title. It is obvious that I cannot cover in any detail the numerous scientific contributions of this period, not even all those which were judged significant at the time. They are too many. After all, for the main scientific center of Europe, the opposite would have been catastrophic! There is hardly any subject of theoretical high energy physics which has not been touched upon by some member or long-term visitor of the Division. As I shall show presently, several contributions were of fundamental importance, although, of course, the selection which follows is entirely my own. I have distinguished two rather vast areas, weak interactions and symmetries on the one hand and dynamical models for hadronic reactions on the other. Most of the work was done on these two subjects, but I shall also mention some very significant out-of-the-main-stream contributions.

I have already presented in the last section, the important work that was done in the Division in the domain of weak interactions by d'Espagnat, Prentki and Cabibbo. Maybe here is the right place to complete the story I left unfinished in Section III. The object of the game was to determine the weak current. Here to determine means to identify it with a known operator of the strong interactions. As we saw, C.V.C. solves the first half of the problem. The weak vector current is identified with the current of isospin symmetry. The identification of the axial part was more subtle: Naïvely, one would expect an extension of the symmetry of strong interactions from $S U(2)$ to chiral $S U(2) \times S U(2)$. The trouble is that there is no obvious trace of such a symmetry in the hadron spectrum. Nucleons do not appear to have degenerate, or almost degenerate, partners with opposite parity. The answer is called P.C.A.C. (Partially Conserved Axial Current) and it involves the concept of spontaneous symmetry breaking. Several names can be associated with it and include M. Goldberger, S. Treiman, Y. Nambu and $\mathrm{J}$. Goldstone. The conclusion is that strong interactions do obey an approximate chiral $S U(2) \times S U(2)$ symmetry but the axial part is spontaneously broken with the pions being the corresponding Nambu-Goldstone bosons.

The final act in the classical weak interaction play marks simultaneously the beginning of a new era. It refers to the building of the first neutrino beams in the early sixties. Until then, decays of unstable particles and nuclei provided the only available tools for the study of weak interactions. All these are low energy and low momentum transfer processes. The construction of high energy neutrino beams, which turned out to be a technological wonder, opened new, in principle unlimited, horizons. It gave rise to the first full-scale CERN-Brookhaven competition, which is a very interesting story by itself. CERN developed a superior 
engineering project but lost the physics part and this failure had a bad influence on the moral of the organization. On the scientific side, the first neutrino experiments proved the separate identity of the second neutrino and established the individual electron and muon quantum numbers.

Cabibbo's 1963 paper put the final stone in the classical construction of weak interactions. Nothing important happened in this direction during the following years neither at CERN nor abroad. However, two years later, a totally unexpected experimental result opened a new chapter which is not closed yet. A measurement of $K_{\mathrm{L}}^{0}$ decays revealed the existence of the $2 \pi$ mode, in contradiction with the invariance under $C P$. This result, less than ten years after the discovery of parity violation, came as a shock to the international physics community. The reluctance to admit $C P$ violation was such that all early theoretical work on the subject aimed at reestablishing $C P$ conservation by inventing various schemes to explain the $K^{0}$ results. Although CERN theorists played an important role in these investigations, I shall not describe them because they all have been disproved experimentally. Among the names I would have mentioned, had I done a more extensive study, are those of John Bell, Jacques Prentki and Martinus Veltman. Incidentally, I want to point out that even some important contributions by American physicists, like T.D. Lee or L. Wolfenstein, appeared as CERN publications because their authors were visiting CERN. This is but one example of the very active visitor's program of the organization.

The only paper from the Theory Division on the subject of $C P$ violation which has passed the test of time, is a very clear analysis by John Bell which has been presented in a joint report with Jack Steinberger [10]. Bell does not propose a model but gives the most general framework to analyze the data. The essential new result is a set of relations which unitarity imposes among the elements of the mass and decay matrices of the $K^{0}-\bar{K}^{0}$ system. The report, written in Bell's classic style, is considered to be one of the best articles in the subject.

Today, almost thirty years after the discovery of $C P$ violation, the chapter is still open. We do not know whether it is a property of the weak interactions or whether it provides the only manifestation of a new, super-weak interaction.

As I said earlier, the search for an understanding of the nature of the weak current had let theorists to uncover a hidden symmetry of the strong interactions. The study of chiral symmetry became soon a central problem in theoretical particle physics. It coincided with the establishment of the eightfold way and was thus extended to $S U(3) \times S U(3)$.

The extension of $S U(2)$ (isospin) to $S U(3)$ as the symmetry group of strong interactions was not a straightforward one. $S U(2)$ is realized in a simple way with the nucleons belonging to the fundamental two-dimensional representation. The direct generalization would yield a triplet $(p, n, \Lambda)$. In fact, this model was proposed in the fifties by Sakata but it was soon disproved by the data. The correct solution, found independently by M. Gell-Mann and Y. Ne'eman, assigns the eight baryons $p, n, \Lambda, \Sigma^{ \pm}, \Sigma^{0}, \Xi^{-}, \Xi^{0}$ in the eight-dimensional adjoint representation of $S U(3)$ and leaves the fundamental triplet empty. This was probably the reason why it took so long to discover. Physicists were conditioned by isospin 
into believing that the lowest-lying states should belong to the fundamental representation. On the other hand, hadron spectroscopy, which was a very rapidly expanding subject, showed that mesons formed $S U(3)$ octets and baryons formed octets and decouplets. This pattern, together with the absence of triplets and the proliferation of 'elementary particles', led some theorists to wonder whether we were not simply uncovering another layer of the onion, i.e. whether all hadrons were not bound states of few 'elementary' constituents which would form triplets. The 'quark model' was proposed independently by Gell-Mann and George Zweig, an American physicist who was visiting CERN in 1964. He called his constituents 'aces' while Gell-Mann called them 'quarks'. Incidentally, I want to point out that Zweig's papers [11] have never been published. Apparently, this was due to his insistance in submitting them to the Physical Review, contrary to CERN's policy to publish only in European journals.

One of the early calculations using quarks as constituents of hadronic matter is due to Victor Weisskopf, then Director General, and Roger Van Royen [12]. Although the quark model became soon quite popular, the status of quarks themselves varied considerably in the course of time. Through the results from deep inelastic lepton-nucleon scattering, as well as those from large angle hadron collisions, they evolved, from purely mathematical objects, into the realm of 'elementary' particles. Incidentally, one of the main motivations behind Weisskopf's decision to build I.S.R., was the smashing of the nucleon and the discovery of physical quarks. This programme was realized, but in an unexpected way. I.S.R. did support the evidence, first found at S.L.A.C., for the existence of quarks, although it did not produce them as physical particles.

Immediately after discovering $S U(3)$, Gell-Mann proposed the chiral $S U(3) \times$ $S U$ (3) algebra of currents. In fact it was this paper which was published in the Physical Review, while the previous one on the eightfold way remained as a preprint. It was immediately realized that Current Algebra was a very powerful scheme and, for several years, it provided one of the dominant research themes in theoretical high energy physics. Its first, and most important, success was obtained, independently, by Stephen Adler and William Weisberger in 1965. They expressed the weak axial vector coupling in terms of the pion-nucleon scattering amplitude. A masterful application of P.C.A.C. and a direct way to fix the relative scale between axial and vector currents, thus giving experimental support to the idea of chiral symmetry.

One of the most significant papers in the early period of current algebra came from the CERN Theory Division. It is signed by Sergio Fubini and Giuseppe Furlan in 1964 [13]. They proposed a general method to derive sumrules out of the equal time commutators of the algebra. They considered a particular matrix element, they saturated the product of current operators with intermediate states and used spectral integral representations to transform it into a sum-rule. The method is very powerful and it was used extensively. The following year, together with G. Rossetti, they proposed an improved version [14], which made use of dispersion relations and was manifestly covariant. In fact, two methods had been developed to extract physical information out of current commutators: the first was this one and the second, proposed by Steven Weinberg in 
1966, consisted in writing the Ward identities of chiral symmetry in the form of low-energy theorems.

During the late sixties, current algebra became one of the main research subjects of the CERN Theory Division. Numerically the production was quite substantial (I contributed personally to that). But among the few results of some importance that I can think of, one is a theorem due to David Sutherland, a fellow from Scotland, and also to M. Veltman and John Bell [15]. Sutherland was the first to look at the consequences of chiral symmetry in the presence of electromagnetic interactions and he derived a very simple, although unexpected, result: at the limit of exact P.C.A.C., i.e. no explicit breaking of chiral symmetry, the electromagnetic decays of pseudoscalar mesons, such as $\pi^{0}$ or $\eta$, are forbidden. The argument is very simple and depends only on the canonical commutation relations among neutral components of vector and axial currents. This result was embarrassing for two reasons: first, it was in obvious contradiction with experiment. Second, it seemed to be against all naïve expectations coming from simple field-theory models. This second difficulty was not immediately recognized and brings me to an important contribution of the Division, the celebrated Bell-Jackiw paper on anomalies. As far as I know, the first person to worry about the compatibility of Sutherland's theorem with field-theory and current algebra was Roman Jackiw, an American post-doctoral fellow who was spending a year at CERN on leave from the Society of Fellows at Harvard. I remember Jackiw discussing this problem with me and we both asked the advice of Henri Epstein, a French mathematical physicist and expert on field theory. Henri explained to us, using a very sophisticated language, that naïve consequences of classical equations of motion and symmetries in field theory are not necessarily correct at the quantum level. I was satisfied with this explanation which involved singular products of distributions and all the associated mathematical artillery, but Jackiw was not. Especially when he became aware of an old calculation by Jack Steinberger who, in 1948, had computed the $\pi^{0} \rightarrow 2 \gamma$ decay width in the one-loop approximation and had obtained the correct answer. Steinberger had used a model in which the pion was a nucleon-antinucleon bound state and had assumed a pseudoscalar pion-nucleon coupling. It turns out that the one-loop diagram, although linearly divergent by power-counting, is in fact convergent once gauge invariance is imposed and the trace over $\gamma$-matrices is performed. Jackiw, discussing with Bell, realized that Steinberger's model was essentially identical with the $\sigma$-model, which was considered as a field-theory example of current algebra. Now the contradiction was clear: on the one hand we had a model which was supposed to reproduce all the results of chiral symmetry, including Sutherland's theorem; on the other we could compute explicitly in perturbation theory and find a non-zero answer. Bell and Jackiw wrote a remarkable paper [16] which contains two parts: in the first they set the problem in a clear and unambiguous way. They compute the one-loop diagram using Pauli-Villars regulators, the only gauge invariant scheme known at that time, and they correctly identify the origin of the problem with the auxiliary Pauli-Villars regulator fields whose masses break the conservation of the axial current. The second part, although incorrect, is also very interesting. They attempt to construct a modified Pauli-Villars reg- 
ularization which would guarantee the simultaneous conservation of both gauge and chiral symmetries. They were the first in a long series of unsuccessful attempts to regularize the anomaly away. This paper, which was soon followed by a similar but more complete one by Adler, established the concept of anomalies in gauge theories, which proved to have deep and far-reaching consequences in theoretical high energy physics. A few years later,in 1971, Bruno Zumino, who had already joined CERN, and Julius Wess from Karlsruhe, found a set of consistency conditions which determine the form of the possible anomalous terms in any gauge theory [17]. The importance of this paper grew considerably over the years. It was originally recognized as a very useful method to discriminate among various anomaly calculations. However, with the introduction of powerful mathematical techniques to the study of the anomaly structure of gauge theories, the Wess-Zumino consistency conditions became the starting point of a new field of research, that of topological field theories.

The last paper I want to mention in the domain of weak interactions and symmetries is a 1969 Nuclear Physics article by David Gross and Christopher Llewellyn Smith [18]. They generalized, to the case of neutrino-nucleon deep inelastic scattering, a sum-rule previously obtained by Curtis Callan and David Gross for electroproduction. In particular, they discussed the structure function which corresponds to the vector-axial-vector interference term which is absent for electron scaterring. These sum-rules played an important role in the development of the parton-model ideas and the eventual emergence of quantum chromodynamics.

There have been a couple of additional seminal papers using current algebra ideas from the TH Division, but they belong to the pre-history of the Standard Model and I shall review them in the next section. In a more complete study, one should have mentioned some of the attempts at constructing a relativistic generalization of $S U(6)$, i.e. at finding a symmetry group containing, in a nontrivial way, Poincaré and internal symmetry. However, today we know of the futility of all these attempts, so I shall not pursue this subject any further.

Let me now come to the second major subject, that of the study of dynamical models for strong interactions. As I said earlier, a very general approach was developed during the fifties which was based on the analyticity properties of the scattering amplitude continued analytically in the complex energy plane. The first dispersion relations were written in the United States between 1955 and 1957. Soon after, there appeared two very fundamental contributions. They gave the subject a tremendous impulse and made it the most promising approach for the understanding of hadronic reactions. The first is due to Stanley Mandelstam who had just joined the University of California at Berkeley (in fact his first paper was written while he was visiting Columbia). Mandelstam extracted from the square diagram in $\varphi^{3}$ field theory the analyticity properties of the two-particle elastic scattering amplitude as a function of two complex variables, energy and momentum transfer. He made two important assumptions: first, one of 'maximum analyticity' which means that the only singularities of the amplitude are those imposed by unitarity and crossing symmetry and second, an assumption about a polynomial boundedness of the amplitude in any complex direction in 
energy and/or momentum transfer. These two assumptions allowed him to write a double dispersion relation, the so-called 'Mandelstam representation', which became the bible for hadronic physics. Partly motivated by this work, Tullio Regge, a mathematical physicist from Italy, published in 1959 and 1960 two papers in which he introduced the fundamental concept of complex angular momentum. If the continuation in the complex plane seems natural for a continuous variable, such as energy or momentum transfer, the same idea applied to angular momemtum, which can take only descrete values, was a quite revolutionary one. In fact, in such a case the uniqueness of the continued analytic function is not guaranteed by the standard theorems. Regge studied the non-relativistic model of potential scattering where he could construct the resulting analytic function in the complex $J$-plane explicitly. As far as I know, the argument for the general case, which is based on an assumption about the asymptotic behaviour of the amplitude for large $J$ and uses a mathematical theorem by Carlson, was first pointed out by André Martin. One of the important practical results of Regge theory was the idea that the asymptotic behaviour of the scattering amplitude for large values of the energy could exhibit a non-trivial dependence on the momentum transfer.

The Mandelstam representation on the one hand and the Regge behaviour on the other, became soon the corner stones of a very ambitious proposal, known as the analytic $S$-matrix theory. Its main advocate was Geoffrey Chew from Berkeley who, together with Steven Frautschi, expressed the set of rules which were supposed to determine directly the elements of the $S$-matrix for any hadronic reaction without appealing to an underlying dynamical model such as field theory. This approach, which from exaggerated heights of faith has fallen into totally unjustified depths of oblivion, gave rise to many fundamental concepts in high energy physics in the development of which the CERN Theory Division played an important role. The work done was in two parallel directions. The first was mathematical and aimed at a rigorous derivation of the somehow vague postulates of analyticity and asymptotic behaviour, starting from the axioms of quantum field theory. The second was directed towards phenomenology and comparison with experimental data.

In the first direction I want to present the work of Jacques Bros, Henri Epstein and Vladimir Glaser on the one hand and André Martin on the other. Glaser was the central figure of mathematical physics at CERN. As a young visitor in Göttingen he had participated in the elaboration of the LSZ (Lehmann, Symanzik, Zimmermann) programme. (In fact, the third paper in the series appeared as GLZ, where G stands for Glaser). Already around 1960, Glaser had started thinking about the problem of analytic completion in quantum field theory. In 1961, he lectured on this subject in Paris. Jacques Bros and Henri Epstein were interested in the same problem and Glaser invited them to CERN. This initiated a long lasting and very fruitful collaboration. Their first joined paper, published in 1963, is considered a classic [19]. In the framework of the LSZ axioms, they derived an analyticity domain for the four-point function, considered as a function of six complex variables (energy, momentum transfer and four mass variables), which contains a vicinity of the physical region. In the LSZ formalism the $S$-matrix elements are obtained by Fourier transforming certain 
vacuum expectation values of products of field operators. Several such functions can be constructed, such as retarded, advanced, time-ordered etc. Causality implies well-defined support properties for these functions in position space, which in turn translate into a primitive analyticity domain in momentum space. Moreover, these Fourier transforms coïncide in certain real regions. Therefore, by the edge-of-the-wedge theorem, they are all boundary values of a unique analytic function. Bros, Epstein and Glaser in their 1963 paper proved that the analyticity domain for the four-point function is larger than the primitive one and contains the physical region. For the first time in physics they used very sophisticated geometrical techniques of analytic completion borrowed from the theory of functions of several complex variables. In 1965, they completed this result by proving the crossing property always for the four-point function [20]. They showed that any couple of physical regions in the $(s, t, u)$ space corresponding to scattering amplitudes involving two incoming and two outgoing stable particles with arbitrary masses, are connected by a certain domain of analyticity.

Bros, Epstein and Glaser studied the so-called 'linear' problem, in which the unitarity condition, a non-linear relation, is not used. Originally unitarity was used in order to control the magnitude of the scattering amplitude. The oldest published example I have found is an article by T.D. Lee in a CERN report of 1961. He argued that the lowest order Fermi theory of the weak interactions must break down at some energies because the cross section for $e \bar{\nu} \rightarrow e \bar{\nu}$ grows too fast, like $k^{-2}$, although the only contributing partial wave is $J=1$. The best known example is a brillant article by Marcel Froissart, who derived his famous bound also in 1961. However, the most systematic use and the most thorough exploitation of the unitarity relation is due to Andre Martin. Through the sixties and the early seventies he created at CERN the best school for this problem, he introduced several other theorists in it and established himself as the undisputed expert in the field [21]. He has obtained two sorts of results: rigorous bounds, à la Froissart, for the growth of hadronic total cross-sections, as well as limits on the pion-pion scattering amplitude on the one hand, and an enlargement of the analyticity domain of scattering amplitudes on the other. In both fields, his results are among the most powerful known today. His initial programme on the analyticity domain aimed at proving the entire Mandelstam representation from the axioms of field theory. Already in 1959 he gave, in collaboration with John Bowcock, a first derivation in the framework of a potential model [22]. In 1966 he obtained the first results in relativistic field theory. In the linear programme, Bros, Epstein and Glaser had proved dispersion relations for negative momentum transfer $-t_{0}<t \leq 0$. Martin, using the unitarity relation, obtained dispersion relations for $t<|R|$ which includes real positive $t$. For the special case of pionpion scattering, crossing symmetry allowed him to obtain the same result for $t$ inside a domain $D$ containing the real segment $t=-28 \mu^{2}$ to $t=4 \mu^{2}$. For not too high energy the domain contains part of the Mandelstam cuts. In a subsequent paper Martin extended this result and established the largest rigorous analyticity domain. It is essentially through his work that we are convinced today that Mandelstam representation is not derivable from field theory. 
I shall leave the domain of mathematical physics at this point and move to some interesting phenomenological models for strong interactions, which have been developed at CERN. I shall present three of them: Amati, Fubini and Stanghellini's multiperipheral model, Van Hove's work on diffraction scattering and his method on the longitudinal phase space analysis and Hagedorn's thermodynamic model. All three aimed at a description of multiparticle production hadronic collisions which represent the bulk of experimental data. None is entirely successful, hardly a surprising result if one takes into account the simultaneous presence of several competing mechanisms, none of them being clearly dominant except for some reactions and some regions of the phase space.

The so-called 'peripheral' model was introduced in 1960 by Sidney Drell and F. Salzman and G. Salzman. The name comes from the fact that this model assigns a dominant role to the exchange of the lightest strongly interacting particle, the pion, between the incident particles. In a series of papers in 1961 and 1962, Daniele Amati, Sergio Fubini and Antonio Stanghellini, partly in collaboration with Mario Tonin and Luciano Bertocchi, generalized the concept of peripherism to a multi-peripheral model [23]. They gave a theoretical justification to the peripheral model by showing that it corresponds to keeping only the nearest singularities to the Mandelstam representation, the so-called 'strip approximation', an approximation which is justified if the energy is not too high. At higher energies the same analysis yields the multiperipheral model for inelastic reactions which consists in taking a chain of lower energy amplitudes linked together by virtual pions. As a next step, they considered the corrections to the model induced by unitarity. They showed that these correction terms could not be interpreted as coming from the exchange of simple poles in the angular momemtum plane and they were thus led to postulate the existence of Regge cuts. This was considered as an unorthodox suggestion and they had trouble with the referee who did not want to accept this idea.

Van Hove's main scientific interest at CERN was on high energy hadronic collisions. He concentrated in the small-angle diffraction region and in an early 1964 paper he developed the ideas of diffraction scattering as the shadow of inelastic collisions [24]. In 1969 he proposed the so-called 'longitudinal phase space analysis' [25], which is not a detailed dynamical model, but rather a general method to study multiparticle reactions. It is based on the phenomenological input that the largest part of the total cross-section is made out of small transverse momemtum secondaries. Van Hove proposed to separate phase space into a longitudinal and a transverse parts and keep only the longitudinal projection. He explained that this physically amounts to generalizing to many-body processes the distinction of two-body collisions into forward elastic, backward elastic and exchange processes. Although this method involves no fundamentally new concepts of any kind, it has been extensively used by experimentalists and even by many theorists. Surprising as it may sound, Van Hove turned out to be one of the most phenomenologically oriented senior theorists of the Division. Under his influence CERN developed a very strong group for the study of diffractive scattering phenomena. Unfortunately this was not a very good choice. We know to-day that the 'soft' scattering events are not very interesting but,in the sixties the accelerator energies available 
were not sufficient to produce many large transverse momentum events and so the fundamental significance of the latter was not clearly recognized.

Hagedorn's thermodynamic model grew out of his early association with the PS division. As I said earlier, he was in charge of computing the multiparticle cross-sections and distributions. In the absence of any detailed dynamical model, he made the simplest possible assumption, namely that a very complicated interaction may take place in a certain volume $V$, but the probabilities for the different final states are determined by the available phase space. This gave rise to the CERN version of the statistical model which he formulated already in 1959 [26]. Later, when he moved to $\mathrm{TH}$, he continued to work in the same direction. He also used the experimental information of limited transverse momentum for final particles and had the idea to associate it to an upper bound for possible values of temperature [27]. He thus postulated a new form of thermodynamics with a maximum value of temperature of the order of $160 \mathrm{MeV}$, or $10^{12} \mathrm{~K}$. Apart from its obvious astrophysical consequences, the model made also the novel prediction of an exponentially growing hadronic mass spectrum. Both these features, finite limiting temperature and exponential mass spectrum, were met with some scepticism when they were first proposed in 1965. However, later, they found their place in the framework of dual models.

This brings me to one of the most influential papers in the entire CERN publication list, the celebrated 1968 article by Gabriele Veneziano [28]. The reason I shall not go into much detail is that, as far as I remember, Veneziano arrived at CERN from the Weizmann Institute with the manuscript essentially ready, so, although the paper appeared as a CERN article, the entire credit should not go to the Division. In fact, in 1968, Veneziano was at CERN only as a summer visitor. Nevertheless, it is true that CERN theorists did play an important role in the subsequent development of these ideas.

Veneziano's paper has the title: 'Construction of a crossing-symmetric, Reggebehaved amplitude for linearly rising trajectories'. In the traditional approach one starts from a dynamical model, for example field theory, and tries to compute the scattering amplitude. Veneziano did the opposite. He guessed a very special form and postulated it directly at the level of the amplitude. He never asked the question of the existence of an underlying theory which would give rise to such an amplitude. His main example was Hector Rubinstein's toy reaction $\pi \pi \rightarrow \pi \omega$. This process is not physically measurable, and its only merit is simplicity. The separate conservations of angular momentum and isospin imply the description by a single invariant amplitude. Veneziano postulated that this amplitude, considered as a function of the Mandelstam variables $s, t$ and $u$, is given by a ratio of $\Gamma$-functions. This extremely simple form has almost magic properties. It is crossing symmetric by construction, the singularities of the $\Gamma$-functions reproduce a spectrum of particles lying on linearly rising Regge trajectories and it has the correct Regge asymptotic behaviour. Most important, it exhibits the property of duality, i.e. the sum of Regge exchanges in the cross channels gives rise to the resonances in the direct channel. This model, which was subsequently generalized to multiparticle amplitudes as well as to particles with spin and isospin, was the base of an entire branch of theoretical physics, that of dual models and 
string theories. Out of these studies emerged new fundamental concepts, such as supersymmetry, infinite dimensional algebras or two-dimensional conformal field theories, whose importance trancends the domain of high energy physics. I shall come back to them in a later section.

This concludes my brief account of the work done in the Division in the two principal subjects, that of weak interactions and symmetries and that of strong interaction dynamics. Before closing this section I want to mention some outof-the-mainstream contributions. I have chosen three subjects: mathematical physics, nuclear physics and foundations of Quantum Mechanics.

Mathematical physics has always been well represented in the CERN Theory Division. In the sixties the permanent staff members in this field were Vladimir Glaser and André Martin. I already had the occasion to present an important part of their work. Here I shall give some additional information.

In 1958, Walther Thirring proposed a field theoretic model involving a massless Dirac field, interacting through a four-fermion contact term, in one space and one time dimensions. The purpose of the model was to represent, in a simplified two-dimensional world, a theory which was strongly advocated by Heisenberg. However, as it has already been pointed out by Thirring, the model, although very simple, had many interesting properties and, over the years, it became a very useful tool in quantum field theory. The main reason is that the model turned out to be exactly solvable and the first operator solution was given the same year by Glaser [29]. It was completed in 1961 by K. Johnson. Glaser gave an explicit expression for the operator $\Psi(x)$ as a functional of the incoming field. He showed that the $S$-matrix is unitary, but it is given by a pure phase, so all non-trivial scattering amplitudes in the model vanish. Thirring's model was the first exactly solvable relativistically invariant quantum field theory and its subsequent study exemplified many interesting phenomena such as two-dimensional bosonization (the model turned out to be equivalent to the Sine-Gordon bosonic model) or the appearance of anomalous dimensions for the operators of a quantum field theory.

The second contribution in mathematical physics that I want to mention comes from $\mathrm{H}$. Epstein and V. Glaser. In a series of papers around 1970 they presented a novel method for the study of the renormalization programme in perturbation theory [30]. They showed by an inductive construction order by order in perturbation series that the only ambiguities in the definition of the $n$-point Green functions are concentrated on the origin in position space and, therefore, they can be absorbed by local counterterms in the Lagrangian. The resulting series satisfies, at each order, the requirements of locality.

Let me now come to nuclear physics. As I have said earlier, this was the most important research subject of the Theory group during the Copenhagen years where the emphasis was on nuclear structure. After 1957 many fellows came to Geneva, so this activity persisted. However, practically no new appointments were made in this field which soon disappeared from the publication list of the Division. The only contribution from this period that I want to mention is the so-called 'cluster model' for nuclear structure [31] proposed in 1958 by Th. Kanellopoulos and K. Wildermuth. The model describes nuclei as aggregates of smaller nuclei and has been quite successful, especially for certain low-lying states. 
After 1960, a new period begun. With the construction of the PS, the main experimental activity for high energy physics moved there. The SC became principally a nuclear physics machine. In fact, for many years, this machine provided the main information about the pion-nucleus interaction, long before the construction of meson factories. I believe that it was Van Hove's suggestion to create a corresponding small group in the Theory Division. Torleif Ericson, a nuclear physicist from Sweden, was put in charge of it. Ericson did not pursue the study of nuclear structure. In collaboration with Magda Ericson, a nuclear theorist from France, they were among the first to study the interaction of pions with nuclei and to explore a regime which was intermediate between the lowenergies of traditional nuclear physics and the new high energies of elementary particles. This decision was a fortunate one. The subject turned out to be quite interesting and allowed them to respond to the expectations of the experimental groups working on the $\mathrm{SC}$ without loosing contact with the rest of the Theory Division. In 1966, M. and T. Ericson published a long article in Annals of Physics which summarizes their early results [32]. Starting from the data on $\pi-N$ scattering and $\pi$ production in $N-N$ collisions they derived a non-local potential for the interaction of pions with finite-size nuclei which takes absorption effects into account and correctly describes multiple scattering phenomena. This article constitutes one of the basic references in this field. In the late sixties, following the developments of current algebra and PCAC in elementary particle physics, the Ericsons applied these techniques to the pion-nucleus interaction [33]. They derived the soft-pion theorems and studied the effects of the extrapolation offmass-shell. I think it is fair to say that over the years the CERN team, enriched periodically with a few fellows and visitors, became one of the strongest in this field worldwide.

I left to the end the subject of the foundations of quantum mechanics. It was certainly out of the mainstream, not only at CERN, but everywhere. In the Division it was the work of a single man, John Bell, who first succeeded in taking it out of the hands of philosophers and bringing it into the world of physics.

I believe that everybody who has ever taught Quantum Mechanics has felt a certain degree of uneasiness concerning its basic axioms. One way to express this feeling is to say that Quantum Mechanics predicts two possible evolutions for a physical system: The first is the usual Cauchy-type evolution described by Schrödinger's equation. The second, more obscure, is the reduction of the wave function when a measurement is performed. The most popular example of this problem is the famous EPR (Einstein, Podolsky, Rosen) experiment. Bell's fundamental contribution to this question was two-fold: First he showed that von Neumann's theorem on the mathematical impossibility of introducing hidden variables in quantum theory was wrong [34]. All the existing proofs, starting from that by von Neumann, had made some additional, and in fact unjustifiable, assumptions concerning the states characterized by such hidden variables. Second, and most important, he showed that the question was in fact a physical one, i.e. it could be answered by experiment. In his famous 1964 paper he derived the so-called 'Bell inequalities' [35]. He showed that no local hidden variable theory could reproduce the quantum mechanical prediction for an EPR-type experiment. 
I think that these two papers, in addition to stimulating exciting experimental work, have completely changed our perception of the profound nature of quantum mechanics.

\section{The rise of the Standard Model}

This section breaks the story. It does not cover any particular period in time, let alone one of the CERN Theory Division. Although it relates the most extraordinary achievement in modern theoretical physics, it is going to be rather short, because the role of CERN in it was only marginal. However, it would have been a mistake to omit it altogether. First because gauge theories changed completely our way of thinking. After 1971, nothing was the same as before. But also, because in science, pleasure and excitement do not come solely by watching the success of one's own ideas. They come mostly from following closely the whole enterprise, from sharing the disappointments and taking part in the expectations.

The road to the Standard Model was a series of misunderstandings. Never in history have so many missed so much for so long. Essentially all the important milestones went unnoticed when they were first proposed. Many important ideas had to be rediscovered again and again. The protagonists did not seem to be aware of each other's, and occasionally even of their own, work. Among the numerous conclusions a historian of science can draw from the study of this period, a very astonishing one is the ultraspecialization of the high energy theoretical physics community. In most Universities worldwide, young researchers were inadequately trained in quantum field theory, let alone statistical mechanics. I remember a remark I made when I passed my thesis: I told the committee that the only things I was really required to know, were how to compute tree-level Feynman diagrams and how to read the Rosenfeld tables. I was only slightly exaggerating!

The first milestone was the construction of non-abelian gauge theories by C.N. Yang and R.L. Mills in 1954. They explicitly wrote the covariant derivative and the curvature tensor for $S U(2)$. They wanted to apply these ideas to isospin, although their detailed motivation is not clear. In the paper they have some remarks concerning global symmetries and locality which I do not understand, but I believe that they were attracted by the aesthetic value and the high degree of symmetry of the theory. To say that this is one of the most important papers in particle physics, sounds today like a trivial statement, but it was certainly not recognized as such at the time. Although it did stimulate a certain amount of interest, it appeared to be plagued by massless vector bosons and nobody knew what to do with them. In this connection, there is a very interesting correspondence between Yang and Wolfgang Pauli who feared that the infrared behaviour of these theories would be untractable. This question of the infrared singularities continued to confuse people even after the proof of renormalizability. It was finally settled by T. Kinoshita and A. Ukawa who proved in 1976 that Yang-Mills theories give a perfectly well-defined field theory at the Green function level. Singularities arise only when one attempts to go on mass-shell. 
I cannot resist mentioning at this point an intriguing and essentially unknown paper by Oscar Klein, who in 1938, wrote something which looks close to a YangMills theory for the interactions between nucleons. The trouble is that he has made some crucial although trivial mistakes in elementary group theory and, even today, it is very hard to judge its real significance.

During the late fifties and the early sixties, there appeared a series of papers which, following the intermediate vector boson hypothesis, attempted to use massive Yang-Mills theories for weak interactions. Julian Schwinger considered an electroweak theory based on the group $S U(2)$ with the photon being the neutral boson. Sidney Bludman wrote an $S U(2)_{\text {weak }} \times U(1)_{\text {e.m. }}$ model in which all weak currents, charged as well as neutral, were left-handed. The most interesting contribution of this period comes from Sheldon Glashow who first understood that, in an $S U(2) \times U(1)$ theory, the photon is not necessarily the vector boson of $U(1)$. It is in general a superposition of $U(1)$ and the neutral component of $S U(2)$. In this way he introduced a mixing angle, the celebrated $\theta_{\mathrm{w}}$. It is amusing to notice that, at the same time, he made all sorts of wrong claims concerning the renormalizability of massive Yang-Mills theories. They were due to mistakes in field theory, which is surprising from a student of Schwinger's and shows the extent of unfamiliarity of particle physicists with the intricacies of quantum field theory.

I leave the story of Yang-Mills theories at this point in order to start another one. To the best of my knowledge, so far CERN theorists had not worked in this field. The new story is that of spontaneous symmetry breaking. It entered the world of particle physics through the works of Y. Nambu and J. Goldstone. By the middle sixties everybody had accepted the idea that chiral symmetry was spontaneously broken with the pions being the near-Goldstone bosons. Completely unnoticed went a work of Philip Anderson, who in 1962, studied the phenomenon of spontaneous symmetry breaking in the presence of long-range forces. But at that time nobody in high energy physics was reading papers in statistical mechanics. Two years later Robert Brout and François Englert fully developed this idea in the framework of a relativistic field theory. They showed that in a spontaneously broken gauge symmetry the vector gauge bosons acquire a mass and the Goldstone bosons decouple and disappear. The resulting theory has massive vector bosons and at least one physical scalar field. The same conclusion was reached independently by Peter Higgs and also by T.W.B. Kibble. Again, there was no immediate reaction. I remember Brout giving a seminar at CERN in 1966 or 1967. I did not understand much of it, but I discovered that I was in good company. One of the reasons is that we were all focussed on strong interactions. If I remember correctly, Brout was trying to apply this idea to the breaking of $S U(3)$.

In 1967 Weinberg's paper appeared in Physical Review Letters. He took over Glashow's model of $S U(2) \times U(1)$ and applied the idea of spontaneous symmetry breaking. The result was revolutionary; for the first time weak and electromagnetic interactions were treated on equal footing. The breaking of the symmetry was responsible for their apparent different strengths as well as for their mixing. The Glashow angle was not any more an assumption but a natural consequence of the model. The paper was clear and beautifully written, still it passed unno- 
ticed. It was presented in our study group at CERN, I believe by Bruno Renner. We unanimously decided that it was uninteresting. I promptly forgot everything about it. There were two reasons for this: First, Weinberg did not have any proof for the renormalizability of his model. Second, as was already mentioned in the title, it was a model for leptons only. Its extension to hadrons seemed impossible. We were all too much attached to the universality of weak interactions and we considered the mere suggestion to abandon it as sacrilegious.

Let me interrupt here in order to present some of the early CERN contributions. I have chosen two of them.

Around 1966, Veltman was trying to understand the deeper origin of the conservation, or near conservation, of the weak currents. In particular, he tried to throw some light on the general confusion which prevailed at that time concerning the so-called 'Schwinger terms' in the commutators of two current components. While he was on a visit from CERN to Brookhaven, he wrote a paper in which he suggested a set of divergence equations which generalized the notion of the covariant derivative of quantum electrodynamics [36]. This fundamental idea was taken up next year and developed further by Bell [37]. He rederived the divergence equations starting from a formal gauge invariance. Although the emphasis was still on Schwinger terms, the underlying gauge principle was explicitly stated. For Veltman this was a clear signal that weak interactions were described by a Yang-Mills theory. Unfortunately for CERN he left that year for Utrecht. His opposition to Van Hove, his former teacher, was legendary and an untarishable source of jokes in the CERN corridors. Senior staff appointments in the CERN Theory Division have often been criticized and Veltman's case is among the most striking ones. He was already at CERN, he probably wished to stay, but Van Hove wouldn't allow it and nobody in the Division was willing to oppose him.

The second contribution of the Theory Division had a totally different motivation. In the late sixties, through the successes of current algebra, a few people started realizing that strong interactions could not provide a cut-off for the weak ones. I believe that the first to make this remark were B.L. Ioffe and E.P. Shabalin from the Soviet Union. To lowest non-trivial order the weak interactions would give quadratically divergent contributions of the form $G \Lambda^{2}$, where $G$ is the Fermi coupling constant and $\Lambda$ the cut-off. This raises the spectre of strangeness and parity violations in strong interactions unless $\Lambda$ is chosen to be very small. From the absence of such violations Ioffe and Shabalin obtained a very low value of $\Lambda$ of the order of a few GeV. This was quite disturbing because the cut-off is supposed to give the energy scale up to which the theory can be trusted. The cure to this disease was found at CERN by Claude Bouchiat, Jacques Prentki and myself in 1968 [38]. We proved that, under the assumption that the chiral $S U(3) \times S U(3)$ symmetry breaking term in the hamiltonian transforms like the quark mass term, the operator which multiplies the leading divergences is diagonal, i.e. it does not connect states with different quantum numbers, strangeness and/or parity. This settled the question of the leading divergences. However, the next-to-leading ones turned out to be equally troublesome. To $n$-th order they are of the form $G\left(G \Lambda^{2}\right)^{n-1}$ and they can contribute to the $K_{\mathrm{L}}^{0}-K_{\mathrm{S}}^{0}$ mass 
difference or to processes like $K_{\mathrm{L}}^{0} \rightarrow \mu^{+} \mu^{-}$. From the experimental point of view, it is as unacceptable to have these processes as ordinary first order weak transitions as to have parity and strangeness violations in strong interactions. Trying to cure this second disease, Sheldon Glashow, Luciano Maiani and myself were led to the introduction of the charmed quark, but again it was after we had left CERN.We proved that weak interactions can be formulated as a Yang-Mills theory even in the hadronic sector. Unfortunately, nobody, not even Weinberg, brought Weinberg's 1967 paper to our attention.

In the meantime several people were studying Yang-Mills theories from a fieldtheoretic point of view. The correct Feynman rules were obtained for the massless case by R.P. Feynman, B.S. De Witt, L.D. Faddeev and V.N. Popov and S. Mandelstam. But the most important work was done by Veltman who studied the divergence structure of the massive theory. He was the first to realize the vital significance of the Ward identities and the importance of working on-shell. In 1971, the world learnt of a student of his, Gerard 't Hooft, who proved the renormalizability of the spontaneously broken theory and the unitarity of the resulting $S$-matrix. This was the turning point. From this moment gauge theories stormed particle physics.

The final touch to the electroweak theory was made the following year. Strictly speaking Weinberg's model for leptons is non-renormalizable because the Adler-Bell-Jackiw anomalies spoil the Ward identities for the axial current. Claude Bouchiat, Philippe Meyer and myself realized that adding the quarks resulted into a cancellation of the anomalies thus removing this final obstacle. A consistent, renormalizable theory of the electroweak interactions was, finally, at hand.

Let me here pause again in order to present the post-1971 CERN contributions. I shall only mention two which refer to the construction of the model. The first is a variation, published by Jacques Prentki and Bruno Zumino in 1972, which did not have neutrino induced neutral currents [39]. Since it was disproved by the Gargamelle experiment the following year, I shall not comment on it any further. The second is a very interesting analysis by Christopher Llewellyn Smith in 1973 [40] who asked himself the following question: in the spontaneously broken phase, when everything is said and done, the theory has only massive particles. By naive power counting it is hopelessly non-renormalizable. However, we know that its $S$-matrix is calculable. How can one see this property without knowing anything about the underlying gauge symmetry? And, second, are there any other theories with a similar hidden renormalizability. In order to answer these questions he used the concept of 'tree-unitarity', which says that the $S$-matrix elements of a renormalizable theory in the tree approximation, considered as functions of the incident energy, cannot grow faster than a certain power of the energy. If this condition is not fulfilled the theory is non-renormalizable already at the one-loop level. The important result, which was generalized later by J.M. Cornwall, D.N. Levin and G. Tiktopoulos, is that any Lagrangian, of dimensions less or equal to four, involving scalar, spinor and vector fields, satisfies tree-unitarity, only if it is equivalent to a spontaneously broken gauge theory, with the possibility 
of adding arbitrary mass terms to vector fields associated with invariant abelian subgroups.

I come now to the second branch of the standard model, i.e. quantum chromodynamics, the gauge theory of strong interactions. Contrary to the electroweak model, it had a very precise experimental motivation. SLAC's famous results on deep inelastic electron-nucleon scattering, followed by similar ones at CERN and elsewhere, prompted theorists to formulate the concepts of the parton model, which is based on two assumptions: Nucleons are bound states of elementary constituants, called partons. At very short distances partons interact with the virtual photon as free, point-like particles. In 1970, H. Leutwyler and J. Stern from CERN showed that the parton model results concerning the structure functions for deep inelastic scattering, can be obtained if one assumes that the free-field singularities dominate the commutator of two current operators in the vicinity of the light-cone [41]. It was one of the first attempts to introduce field-theoretic ideas to the parton model and played an important role in the final development of quantum chromodynamics.

The first person to discover the property of asymptotic freedom was Gerard 't Hooft who announced it at a meeting in Marseille in 1972. It was presented as a property of Yang-Mills theories with unbroken gauge symmetry. The physical theory of quantum chromodynamics was proposed the following year by H.D. Politzer as well as D.J. Gross and F. Wilczek

\section{Beyond the Standard Model}

This is the last section. It is supposed to cover the period after 1971. In fact, I shall be extremely brief and general for the last ten or twelve years. This should not be interpreted as a value judgement. I have a very high respect for the work done at present in the Division. However, my rule, up to now, has been to give the general trends and single out only the individual contributions which have become part of our scientific heritage. I am unable to make such a selection among the papers published in the eighties. There are two reasons for that: the obvious one is that the lapse of time is too short and I am getting too old and out of touch. But there is a more profound one. For reasons upon which I shall comment later, theoretical high energy physics has become increasingly abstract and mathematical and further removed from experimental data. I believe that it is impossible to tell right now which, if any, of our present speculations, which are very interesting in their own right, will be incorporated into the physics curriculum of the twentieth first century. However, if I had to, I would bet on the mathematical techniques, not so much on the detailed physical models. I believe that geometry and topology, the natural languages of gauge theories, have come to stay. If the sixties forced the average physicist to learn elementary group theory, the seventies and the eighties did the same for differential geometry and topology.

As I said earlier, gauge theories changed radically our way of thinking. A historian going through the literature of the early seventies, may get the mistaken idea that nothing had happened. The scientific journals are not saturated with 
papers on gauge theories. However, this conclusion is wrong for several reasons. First it ignores the obvious fact that the papers published at a given moment represent usually projects which had started several years earlier. Second, and most important, gauge theories represented a real break with respect to our old ideas and prejudices. There were so many new things to learn, so many new techniques to master. If I remember correctly, most people were aware of the revolution that was taking place, although few had the courage to join the fun immediately.

Before going into the work of the Theory Division, let me again present the main theoretical ideas which dominated high energy physics after the standard model.

The first direction was, naturally, the study of the physical consequences of the model and their comparison with experiment. It was a long programme which went through several stages: in the early seventies the aim was to discriminate among the various possible models. The emphasis was on yes-or-no experiments, like the existence of neutral currents, that of charmed particles, heavy leptons, parity violation in atomic physics, etc. As the experiments progressed, more quantitative questions could be asked, but it was only quite recently that the entire model could be tested at the level of radiative corrections. A parallel approach has been followed concerning quantum chromodynamics. The first evidence for a $Q^{2}$-dependence of the structure functions was found in the early seventies, but it took almost twenty years of strenuous efforts in deep inelastic lepton scattering, jet production experiments, studies of charmonium and $\Upsilon \Upsilon$-systems and, finally, the hadronic decays of $Z^{0}$, before the full two-loop calculations could be meaningfully compared with the data. The amount of theoretical work that has been invested in these studies has been quite impressive, second only to the unprecedented experimental effort.

The second direction of theoretical studies is commonly described by the title 'beyond the standard model'. The motivation is very clear. Gauge theories have opened new horizons in the theory of the fundamental interactions. Many old problems were solved, only to make room for new and more profound ones. Questions, which could not be meaningfully addressed before, became now liable to scientific investigation. This extraordinary theoretical drive can be summarized by two words: more symmetry. Everytime a new problem appeared physicists reacted by searching for a model with higher symmetry. According to one's taste or prejudice, this process can be viewed either as a search for a truly unified theory of all interactions, or as an endless series of 'fuites en avant'. I shall try to give the main steps:

The standard model is not a unified theory. It is based on the group $U(1) \times$ $S U(2) \times S U(3)$ and each factor brings a new coupling constant. What is worse is the presence of the abelian factor $U(1)$ because $U(1)$ admits any number of coupling constants. In a non-abelian group the coupling constant is fixed by the gauge boson self-coupling and it must be the same, apart form Clebsh-Gordan coefficients, for every matter multiplet. For $U(1)$, however, this is not so, in other words, the standard model does not explain the observed quantization of electric charge. Faced with this problem, physicists took the first step towards higher 
symmetry. The hypothesis of grand unification states that $U(1) \times S U(2) \times S U(3)$ is the remnant of a larger, simple group $\mathrm{G}$, which is spontaneously broken at very high energies. This idea was first proposed in 1974 by Howard Georgi and Sheldon Glashow who considered the group $S U(5)$. Other groups, such as $S O(10), E_{6}$, etc., have also been studied. Using the renormalization group equations, one can estimate the order of magnitude of the unification energy which turns out to be larger than $10^{15} \mathrm{GeV}$. A very important consequence of grand unified theories is the non-conservation of baryon number, and this has triggered a very intense experimental effort to detect a possible proton decay.

Grand unified theories are aesthetically attractive and solve the problem of electric charge quantization. However they raise a new one: they involve two widely separated energy scales, the super-heavy breaking occurs at energies above $10^{15} \mathrm{GeV}$, while the standard model one at $10^{2} \mathrm{GeV}$. The only way to implement a spontaneous symmetry breaking using the available field-theory technology, is to introduce appropriate scalar fields. However, scalar fields have a tendency to acquire, through higher order corrections, masses equal to the largest available mass scale. This means that grand unified theories cannot sustain, in a natural way, the two energy scales. The light breaking tends to join the heavy one. This is known as the 'gauge hierarchy problem'. Once more theorists tried to increase the symmetry of the model. It was easy to see that going to higher gauge groups did not help. All grand-unification groups suffer from this disease. What was needed was a symmetry which would keep scalar fields naturally light, in other words a symmetry which would make scalar fields behave like fermion fields were known to behave. This led to the idea of a fermi-bose symmetry, or supersymmetry. Such a scheme was found in two-dimensional models by Jean-Loup Gervais and Bundji Sakita and was introduced in four-dimensional field theories by Julius Wess and Bruno Zumino in 1973 [42].

Supersymmetric grand unified theories have been constructed and their properties have been extensively studied. It turns out that supersymmetry must survive at sufficiently low energies and its consequences should be detectable in the near future. A rich spectroscopy of new particles is predicted. The new problem arises now at the high energy front. The presence of new particles makes the unification energy higher, of the order of $10^{17} \mathrm{GeV}$. This is too close to the so-called 'Planck scale' of $10^{19} \mathrm{GeV}$, which means that we can no longer neglect gravitational interactions. This opens a completely new chapter. Particle physicists had ignored gravitation which had resisted all attempts to quantization. Supersymmetry offered a new line of approach. The anti-commutator of two global supersymmetry transformations is a space-time translation. Therefore, gauging supersymmetry implied the gauging of translations, in other words local supersymmetry would include general relativity. It was hoped that the remarkable convergence properties of supersymmetric quantum field theories would fianlly win and yield a consistent quantum gravity. The dream of a trully unified gauge theory of all fundamental interactions would have been realized. The simplest supergravity theory was constructed in 1976 by Sergio Ferrara, Daniel Friedman and Peter Van Nieuwenhuizen. It was a toy model containing only the graviton and its superpartner, the gravitino. The candidate for a real theory, the 
supergravity with eight supersymmetry generators, was constructed and studied in 1978 by Eugène Cremmer and Bernard Julia. The $N=8$ supergravity was the end of a road, the road to Symmetry. No field theory with higher symmetry is known to exist. Therefore it was either the sought for solution, or the entire approach would have failed. Its perturbation theory was studied in detail. For several years people hoped for a miracle to happen. It did not. By the early eighties all were forced to admit that $N=8$ supergravity remained a hopelessly non-renormalizable theory. The road to Symmetry appeared to lead to a dead end. This failure was interpreted as a failure of local quantum field theory. The very notion of a local field, which was inherited from the notion of a point particle in classical mechanics, had to be abandoned. This concept had been challenged several times in the past and people had often tried to construct theories of extended objects. However, it was only recently that the motivation for such a radical step appeared to be compelling. Strings are the simplest extended objects. Although theories of higher dimensional objects have been studied (membranes, etc.), only strings seem to yield consistent theories. They use the concepts and techniques developed for the dual models, but the physical meaning has changed. The fundamental length is no more of hadronic scale but of Planck scale: not $10^{-13}$ but $10^{-33} \mathrm{~cm}$. Joel Scherk has played an important role in this conversion. In 1974, in collaboration with John Schwarz, he proposed to interpret the spintwo zero-mass state which appeared in the spectrum of the dual models as the graviton, and in 1976 together with Eugène Cremmer, he used the Kaluza-Klein mechanism of dimensional compactification in order to arrive at four-dimensional theories. The connection with the theory of two dimensional gravity, a central theme in many recent developments, can be traced to the work of A. Polyakov in the early eighties and the recent revival of the theory is due to Michael Green and John Schwarz who, in 1984, constructed the first anomaly-free superstring theories.

For somebody who is used to local quantum field theory, the obvious generalization to a theory of quantized strings would be to consider a theory in which the fields, instead of being operator-valued functions (in fact, distributions) of the space-time point $x$, they would be operator-valued functionals of the string function. We could call such a theory 'quantum field theory of strings'. Nobody has succeeded in writing such a theory and we believe today that it can not be written using the available mathematical technology. Let me note in passing that one of the most attractive features of string theory to the eyes of many theoretical physicists, is precisely the fact that it makes contact with the most advanced research in modern mathematics. The approach which has been followed in string theory corresponds to a first quantized theory and it is the generalization of particle mechanics. The classical mechanics of a freely moving relativistic string can be obtained by extremizing the invariant area of its trajectory which is a two-dimensional world-surface. Let its points be parametrized by $X_{\mu}(\sigma, \tau)$. The index $\mu$ runs form 0 to $d-1$, where $d$ is the dimensionality of the embedding space in which the string is moving. On the other hand, $X_{\mu}$ can be viewed as an ordinary field in a two-dimensional space. It is this equivalence between string theory and quantum field theory in $1+1$ dimensions that helped to make progress 
in the theory of quantized strings and established a connection with the statistical mechanics of two-dimensional critical phenomena. A most remarkable result is that $d$ can not take arbitrary values. Assuming the ambiant space is flat, one can construct a consistent theory of superstrings only if $d=10$. Hence the need to consider the possibility of compactifying the six additional dimensions and study the properties of the resulting theory. The problems one encounters in this programme, both mathematical and physical, are formidable and at present it is too early to accurately evaluate the significance of these ideas. However, I want to emphasize that superstring theory is today the only candidate for a consistent theory of quantum gravity. Furthermore, at least one of its ingredients, namely supersymmetry, can be tested experimentally in the near future.

Let me now come to the contributions of the CERN Theory Division to this world effort. I shall start with the first direction, the physics of the standard model. For reasons of presentation I shall distinguish two periods, before and after 1976. The separation date has not been chosen randomly. Believing in gauge theories in 1972 required an act of faith. Above all, faith in field theory, and most theorists had learnt to mistrust field theory. By 1976, on the other hand, gauge theories had passed many experimental tests. The study of their physical consequences became the dominant research theme everywhere. The CERN Theory Division was no exception.

In the early seventies, the CERN papers on gauge theories are very rare. I have already mentioned the Prentki-Zumino model and Llewellyn Smith's work on the high energy behaviour of weak interaction amplitudes. In fact, in 1972, the Division had seventeen staff members (nine permanent and eight fixed term). The only one among them, who studied the phenomenological consequences of the standard model, was Llewellyn Smith. His 1974 article on the properties of neutral currents [43], written in collaboration with Dimitri Nanopoulos, was the first complete study in this new and exciting field. This lack of interest, coming from the theorists of a laboratory whose first claim to fame was precisely the discovery of weak neutral currents, is hard to understand. I shall come back to this question in the Conclusion because I believe that it reflects quite accurately the Division's weakest point, namely its slow response to this revolution. During the same period Fermilab had developed a very active group of gauge theory phenomenology. Let me notice, however, that some interesting contributions came from visitors and fellows. I shall present briefly a few of them: one of the first complete one-loop calculations in the new renormalizable electroweak theory is due to W.A. Bardeen, R. Gastmans and B. Lautrup and appeared in 1972 [44]. They computed several static quantities of the vector bosons and the fermions in the model and discussed the gauge invariance of the $S$-matrix elements. There was also a series of papers by C.H. Albright, often in collaboration with C. Jarlskog, on charmed particle production, properties of heavy leptons etc. [45]. Between 1972 and 1974, Gerard 't Hooft was a visitor at CERN. During the same period, Veltman also spent some time there. They published several very important papers on the properties of gauge theories, some of which have opened new fields of research. Together they wrote two papers. The first, presented in the 1972 Marseille meeting, completes the renormalization programme for spontaneously 
broken Yang-Mills theories [46]. The second studies the one loop divergences in the theory of gravitation [47]. In a series of two papers published in Nuclear Physics in 1973, 't Hooft developed the renormalization group algorithm in the scheme of dimensional regularization [48]. The following year he published, again in Nuclear Physics, three fundamental papers: in the first he showed that an $S U(N)$ gauge theory in the limit $N \rightarrow \infty$ is described by planar diagrams only [49]. Numerous studies have been performed since using this idea. In the second he solved such a theory in the special case of a two-dimensional space-time [50]. Finally in the third he constructed his famous magnetic monopole classical solution [51]. He studied an $O(3)$ gauge theory with a triplet of Higgs fields. He showed that there exists a classical solution, which is regular everywhere and corresponds to a unit magnetic charge.

After 1976, the situation changed radically. It was obvious that gauge theories had come to stay. The electroweak model was already taught in all graduate schools and quantum chromodynamics was admitted as the fundamental theory of strong interactions. At CERN several research teams were formed, mainly by the younger members. The one by John Ellis, Mary-K. Gaillard and Dimitri Nanopoulos became soon one of the most active world-wide. Their first paper I want to mention is a 1976 article on the phenomenological profile of the Higgs boson [52], which, for several years, was the guide to experimental search. Between 1977 and 1978, E.G. Floratos, D.A. Ross and C.T. Sachrajda performed a two-loop calculation for the deep-inelastic scattering in quantum chromodynamics [53]. A similar calculation, including the time-like region, was completed in 1980 by W. Furmanski and R. Petronzio [54]. André Martin used his expertise in potential models to derive many rigorous results in charmonium spectroscopy. The discovery in 1977 of the upsilon provided a new system for a more accurate application of these ideas. His review article in Physics Reports, written in collaboration with $\mathrm{H}$. Grosse, summarizes all the results on heavy quark-antiquark bound states [55]. As we shall see presently, this intense phenomenological activity was developed in parallel with other, more abstract, subjects. Together, they made the CERN Theory Division during the late seventies a very exciting and stimulating place.

Let me now come to the second direction, the one I had called 'beyond the standard model'. Here I shall not follow any chronological order, but I shall present the CERN contributions according to the sequence grand unified theories - supersymmetry - supergravity-strings.

The 'standard' grand unified model is $S U(5)$. We know today that it does not fit the data, but it still exemplifies all the essential theoretical ideas. The two fundamental papers on $S U(5)$ are the 1974 Georgi-Glashow one, where the model was proposed, and a second one in the same year by Howard Georgi, Helen Quinn and Steven Weinberg, where the renormalization group evolution of the three physical coupling constants was computed and they were found to meet roughly at the grand unification scale. In 1977, this idea was taken again at CERN by A.J. Buras, J. Ellis, M.K. Gaillard and D.V. Nanopoulos [56]. They made a more complete calculation of the renormalization group functions and, most important, they were the first to examine the evolution of the fermion 
masses. $S U(5)$ predicts the mass ratios of the down quarks and the charged leptons of a given family, but these predictions are valid at the grand unification scale. The CERN team computed the evolution of these ratios at low energies. Their best known result is that the $m_{\mathrm{b}} / m_{\tau}$ ratio agrees with experiment only if the model contains just three fermion families. As I said earlier, an essential prediction of grand unified theories is the non-conservation of baryon number. In $1978 \mathrm{M}$. Yoshimura remarked that this property could be used to explain the observed dominance of matter over anti-matter in the Universe. He was, apparently, unaware of a similar proposal formulated more than ten years before by A.D. Sakharov. In fact, Yoshimura's paper contains some technical mistakes which are absent from the early Sakharov's paper. During 1978 and 1979 several groups corrected and expanded this idea and one of the first to do so was the trio Ellis-Gaillard-Nanopoulos from CERN [57]. These works were quite significant because they contributed to the development of a new field, the application of particle physics to astrophysics and cosmology.

The concept of a symmetry between fermions and bosons was first introduced in two space-time dimensions, in the framework of dual models. Jean-Loup Gervais and Bundji Sakita discovered such a symmetry, which was called 'supergauge symmetry' in the dual model formulations of A. Neveu and J.H. Schwarz and P. Ramond. In four dimensions related ideas had also been studied in the Soviet Union by Yu. A. Gol'fand and E.P. Likhtman as well as D.V. Volkov and V.P. Akulov. However, I think it is fair to say that the enormous impact of supersymmetry to theoretical high energy physics is due to the work of Julius Wess and Bruno Zumino. In fact, supersymmetry occupies a very special place among our fundamental concepts; it has received until now absolutely no experimental support, still it has excercised a tremendous influence in practically every recent development in particle physics.

Wess and Zumino in their 1973 paper introduced the four-dimensional supersymmetry algebra [42]. (In fact, they were still calling it supergauge algebra: the word 'supersymmetry' was introduced by A. Salam and J. Strathdee in 1974). They showed that the algebra closes with the use of commutators and anticommutators and that it contains the algebra of the Poincare group. They also built, by trial and error, the first irreducible representations in terms of field operators, a 'scalar' multiplet containing a Majorana spinor and four scalar fields, as well as a 'vector' multiplet with one vector, two spinors and four scalars. They also found the first rule for a tensor calculus, because they showed how one could multiply two scalar multiplets in order to obtain a third one. Finally they constructed free invariant actions with the scalar and the vector multiplets and showed that, in both cases, some of the components were non-propagating auxiliary fields. It is a most remarkable paper combining technical skills with exceptional intuition. In a second paper [58], they studied a lagrangian field theory model with a self-interacting scalar multiplet. The model consists of Yukawa, $\phi^{3}$ and $\phi^{4}$ couplings and it is renormalizable by power counting. Supersymmetry manifests itself in the form of relations among masses and coupling constants. Wess and Zumino showed that, at the classical level, the Majorana spinor and the two physical scalars have the same mass and all couplings can be expressed 
in terms of a single coupling constant. They computed explicitly the radiative corrections at one loop order and found that these special relations imposed by supersymmetry were maintained. This led them to discover a most peculiar feature of the model: at the order of one loop, there was no mass or coupling constant counterterm. Only a common wave function renormalization was required. I remember Zumino explaining these puzzling results to me during one of my visits to CERN. I could not see any deep reason for these divergence cancellations and I concluded that they ought to be one loop accidents and they should disappaer at higher orders. Nevertheless, we decided to check the two-loop counterterms. To our surprise we found that the cancellation persisted. It took us several weeks to find the general proof to all orders [59]. These non-renormalization theorems made supersymmetry a possible candidate for the solution of the gauge hierarchy problem in grand unified theories.

An important step in the development of supersymmetry theory was the introduction of the superspace formalism by A. Salam and J. Strathdee in 1974. It was completed the same year by Sergio Ferrara, Zumino and Wess [60]. The formalism consists in writting the supersymmetry transformations as generalized translations in an eight-dimensional super-space. A point in superspace is parametrized by an ordinary four-vector $x_{\mu}$ and an anti-commuting complex twocomponent Weyl spinor $\theta_{\alpha}$. Because of the anticommuting character of the $\theta$ 's, a field in superspace (a superfield) is equivalent to a finite number of fields in ordinary space, in other words, this method provides an algorithm for finding field representations of supersymmetry. Furthermore, since the product of two superfields is again a superfield, we have the basis of a complete tensor calculus. One can construct Lagrangians directly in superspace and derive Feynman rules for superfields. These rules have considerably simplified supersymmetry calculations and have uncovered the origin of the non-renormalization theorems.

The final ingredient which was needed before applying supersymmetry to particle physics was the construction of the supersymmetric extension of gauge models. This problem was solved at CERN in two fundamental papers of 1974, the first by Wess and Zumino and the second by Ferrara and Zumino. The first derived the supersymmetric extension of quantum electrodynamics [61]. It used the component formalism and showed that in order to supersymmetrize quantum electrodynamics one should extend the electron field to a full complex scalar multiplet and the photon field to a full vector multiplet. Furthermore, the scalar function of the gauge transformation should also be extended to a scalar multiplet. The resulting theory describes the electromagnetic interactions of the electron and its scalar partners but also Yukawa-type couplings between the electron, its scalar partners and the spinor partner of the photon. In the same paper, the concept of the so-called 'Wess-Zumino gauge' was introduced. In fact, in a general gauge the theory is non-polynomial. Wess and Zumino showed that there exists a partial gauge fixing which puts to zero many auxiliary fields of the vector multiplet and makes the theory polynomial. There is still the freedom to perform ordinary gauge transformations, but they should be compensated by a supersymmetry transformation. The Ferrara-Zumino paper addressed the question of the supersymmetrization of Yang-Mills theories [62]. It used the superfield formalism and 
derived a very simple and interesting result: in the analogue of the Wess-Zumino gauge, a Yang-Mills theory describing the interaction of the gauge bosons with a multiplet of Majorana fermions in the adjoint representation of the group, is automatically supersymmetric.

These two papers opened the way for an application of supersymmetry to particle physics. Pierre Fayet from the Ecole Normale Supérieure in Paris wrote the supersymmetric extension of the standard electroweak model and derived all the phenomenological consequences. His results have been extensively used for the experimental searches of the supersymmetric particles. He often visited CERN and a couple of his papers appeared as CERN preprints. It is too early today to say whether supersymmetry will enter the future editions of the Rosenfeld tables. If it does, it will certainly be CERN's most important theoretical contribution. However, even today we can assert that it has been one of the most influential ideas of the last twenty years.

The two most important supergravity models, the first $N=1$ supergravity and the largest $N=8$ one, were discovered at the Ecole Normale Supérieure in Paris, where also many of their properties have been investigated. Nevertheless, many significant contributions came from the CERN Theory Division regarding both the mathematical structure and the phenomenological applications. Stanley Deser and Bruno Zumino presented in 1976 their formulation of $N=1$ supergravity [63]. They used the first order formalism for gravitation in which the vierbein and the connection are treated as independent fields. It turned out that this simplified considerably the calculations and they were able to show in a simple and elegant way that the sum of the Einstein term and that of a massless minimally coupled Majorana spin 3/2 field described by a Rarita-Schwinger action, is invariant under local supersymmetry transformations. Furthermore the resulting equations of motion were free of the pathologies associated with higher spin fields.

All early formulations of supergravity were written in terms of physical fields. Only the graviton and the gravitino were present. The physical interpretation was unambiguous but the closure of the supersymmetry algebra required the use of the equations of motion. This was not quite satisfactory from the field theory point of view, especially for the implementation of the renormalization programme. The minimum set of auxiliary fields, which guarantees the invariance of the action offshell, was first found by Ferrara and van Nieuwenhuisen at CERN in 1978 [64]. The same authors presented the set of rules for a tensor calculus of supergravity [65]. These rules turned out to be very useful for the construction of invariant actions satisfying local supersymmetry.

One of the outstanding problems in every attempt to build a quantum theory of gravitation is the observed vanishingly small value of the cosmological constant. In perturbation theory this parameter is divergent and one has to fine tune it to zero. Between 1983 and 1984 a particular class of supergravity theories was found in which the cosmological constant naturally vanished without any finetuning of parameters. In these theories the minimum of the super-Higgs potential was equal to zero but it had flat directions which made the vacuum expectation value of the scalar field undetermined, at least in the tree approximation. These 
models were called 'no-scale models' [66]. They were developed in collaborations mainly between the CERN Theory Division and the Ecole Normale Supérieure in Paris. The main contributors were J. Ellis, S. Ferrara and D.V. Nanopoulos from CERN and E. Cremmer and C. Kounnas from E.N.S. In a series of papers the most general no-scale models were constructed and the possibility of determining the minimum of the potential by radiative corrections was examined. The interest for these models has been revived more recently because, as Edward Witten has shown, they may appear naturally in the framework of some superstring theories. They represent the most promising candidates for a realistic effective theory of supergravity.

This brings me to the last development in the road beyond the standard model, the theory of quantized superstrings. It grew out of the dual models which were developed in the late sixties and seventies. As I said earlier, one can distinguish two periods in their history: the early one in which the motivation was the description of hadron physics and the late one which aimed at a quantum theory of gravitation. The transition was gradual extending almost over a decade, but the most active periods were before 1975 for the first and after 1984 for the second.

The CERN contributions to the development of the dual models were very important. Let me comment here briefly on the main steps:

The original Veneziano formula described the four-particle amplitude in an approximation which we would call today tree-order. Obviously, two generalizations were required before one could talk about a complete theory: the construction of a tree-level $N$-point amplitude and the extention to an arbitrary number of loops. Both problems attracted immediately the attention of many theorists worldwide, and the CERN contributions were among the most significant.

The problem of the $N$-point amplitude was practically solved already in 1968 . The first paper on the subject is a Physics Letters article by K. Bardakci and H. Ruegg [67] who were both visiting CERN. As far as I know they were the first to write the $N$-point function as a multiperipheral diagram with the exchange lines represented by Regge families. In their first paper they applied this idea to the five-point amplitude (two incoming and three out-going particles). This technique was subsequently generalized to the six- and seven-point functions and finally to the entire $N$-point amplitude by Chan Hong-Mo and Bardakci and Ruegg [68]. Similar results were obtained independently by C.J. Goebel and B. Sakita.

It took somehow longer to derive the correct form of the multi-loop amplitude. As far as I know, the first to realize that unitarity implied the multiloop generalization was Holger Nielsen from Copenhagen who made a conjecture about the form of the amplitude. The formal solution was developed mainly at CERN by Claude Lovelace [69] on the one hand and Victor Alessandrini and Daniele Amati on the other [70]. These papers are very important, because, for the first time, the $M$-loop function was written as an integral over a surface with $M$-holes. This expansion in the genus of the surface became the standard perturbation series in string theories. 
A second fundamental question in dual models was the problem of unitarity and the absence of ghosts from the spectrum of states. The problem is similar in principle, although much more complicated in practice, with the corresponding one in gauge theories. Let me consider the simplest example of quantum electrodynamics. Gauge invariance, which is associated with the vanishing photon mass, implies the presence of only two helicity states. When quantizing the theory, one has a choice between two strategies: Stick to the physical degrees of freedom, to the expense of manifest Lorentz covariance, or introduce unphysical states in order to ensure covariance at every step. In either case a more or less complicated proof is needed before one could claim that the resulting theory is physically relevant. It was soon realized that string theories had a very large gauge invariance, which can be viewed as the invariance under reparametrizations of the two-dimensional multi-hole surface. Therefore, the crucial problem emerged, whether states of negative norm (ghost states), are present in the spectrum of physical states. The complete answer to this question turned out to be lengthy and rather complex with CERN theorists contributing many important steps.

The first unitarity proof in a covariant quantization approach for the open string model is due to P. Goddard and C.B. Thorn in 1972 [71]. They showed the absence of ghosts for the Pomeron sector, i.e. unit intercept at $t=0$ for the corresponding Regge trajectory, provided the dimensionality of the embedding space was $d=26$ for the bosonic string or $d=10$ for the Neveu-Schwarz one. This result was extended the following year to the closed string by David Olive and Joel Scherk [72]. The second approach, namely the quantization in a transverse, non-covariant gauge, was followed, also in 1972 , by P. Goddard, C. Rebbi and C.B. Thorn who showed the Lorenz covariance of the resulting theory [73]. In this connection, let me mention another important issue, namely the nature of the Pomeron singularity. In constructing higher order dual amplitudes, one obtains, in general, cuts rather than poles. C. Lovelace conjectured that, at $D=26$, the Pomeron cut was reduced to a pole. In 1972, Eugène Cremmer and Joel Scherk established the existence of the Pomeron poles and showed that they had the correct factorization properties [74].

Between 1968 and 1973, the study of dual models was the main research subject in the CERN Theory Division. Around two hundred articles were written but I have chosen only the ones which I consider more important with today's perspective. Another criterion was the strength of the authors' connections with CERN. Before leaving this subject I want to mention one more article which satisfies this criterion only marginally: two out of four authors were at CERN. It is an article by P. Goddard, J. Goldstone, C. Rebbi and C.B. Thorn. Although the relation between dual models and string theories was established quite early, (it goes back to the classic works of Y. Nambu, H.B. Nielsen, L. Susskind, etc. in 1969-1970) most results were initially obtained using the old dual model language. One of the first systematic studies of these models as string theories is due to these authors and it was published in Nuclear Physics in 1975 [75].

With the emergence of quantum chromodynamics, the initial enthusiasm for dual models as the theory of strong interactions gradually disappeared. As I said 
above, some theorists started suspecting that the correct domain of applicability of string theories should be quantum gravity rather than hadronic physics. In this connection, I want to mention a 1977 Nuclear Physics paper by F. Gliozzi, J. Scherk and D. Olive, which appeared as a CERN preprint, although only the third author was at CERN [76]. They studied the Neveu-Schwarz-Ramond superstring model in the low-energy region and showed that it gives rise to a supergravity theory.

The new era of superstring theories started officially in 1984. For several years it dominated theoretical high energy physics research and contributed to the development of many fundamental concepts mainly at the frontiers with mathematical physics and statistical mechanics. Important progress has been made through the cross-fertilization of these fields. Physicists and mathematicians, having seemingly different motivations, have found their work intimately related. Progress towards a covariant formulation of string theories has profited from advances in the theory of two dimensional critical phenomena and, conversely, many string-motivated works on conformal field theory in two dimensions opened new paths in statistical mechanics. In the phenomenology of elementary particles the situation is less clear. String theories seem to restrict the various choices of grand-unified models but the precise connection is still obscure. I believe that the field faces an important challenge: Its future depends upon our ability to make progress towards non-perturbative solutions. As happened with local field theory, all questions which admit a perturbation expansion have, to a large extent, been understood. We are now facing the hard, strong-coupling problems. Their solution requires, most probably, the elaboration of new mathematical tools and, almost certainly, the introduction of new physical ideas. Whether and how this will happen, will determine the real significance of much of the work already done. This is the reason why, as I explained already above, I shall not single out any of the recent work done in the Division. Nevertheless, I want to emphasize that it has often been at the frontier of current research.

\section{Conclusions}

CERN spans forty years of history. Forty years that have shaped our understanding of the physical world. To this understanding the Laboratory has contributed its fair share. We can look at its record from two sides: The first is the wish of the founding fathers, namely the development of high energy physics in Western Europe. Here, the emphasis is on the role of the Laboratory as training center for European physicists. The second is CERN's own research record, its contribution to the creation of new knowledge. Let me examine each point separately for the Theory Division.

I have had already the opportunity to emphasize CERN's contribution to the renaissance of European high energy physics. I believe that, for the Theory Division, the most important element has been its extremely rich visitor's programme. No other place in the world has had such a high ratio of kinetic energy to rest mass. This programme, not only offered post-doctoral positions to generations of young theorists, but also gave them the opportunity to meet many of the most 
influential physicists from Europe and the United States. It is this extremely high concentration which made CERN such a unique place. I do not know who, if anyone, should be credited for this open-door policy, but it played a key role in the fulfilment of CERN's pedagogical mission. Let me give some crude statistics: I asked three of my colleagues, independently, to name thirty leading European high energy theorists between forty and sixty years of age. As it turned out, the lists were, to a large extent, identical. Out of the thirty-six physicists in the combined list, thirty-two had been for at least one year at CERN. And for most, this visit corresponded to their formation years.

The scientific record of the CERN Theory Division was the subject of this chapter. As I have said already, it has published around seven thousand papers. I have tried to mention the ones I considered as most significant. What is the overall picture? It will not come as a surprise to the historian to discover that the performance of European theorists went, by and large, in parallel with that of their experimental colleagues. During the fifties and the sixties the United States excercised a clear dominance over the entire subject. Europe's role followed the rise of the standard model. The CERN theorists have often been blamed for their lack of participation in the construction of the standard model. I consider such a reproach as meaningless. I tried to show that the standard model was discovered mainly by individuals often working in relative isolation. Most of them could not have been hired by CERN anyway. Furthermore, the same reproach could be addressed equally well to most other institutions in the world. What I find more disturbing is the Division's failure to respond promptly after the discovery. Revolutions are always made by very few. Most CERN theorists were slow to realize that one was taking place. The first failure can be attributed to bad luck. The second shows a certain bias in the hiring decisions. The obvious reason is that the overwhelming majority of the senior members were not interested in weak interactions and they did not hire younger members who were. This unbalance was later corrected. After the early seventies, the entire Laboratory, experimentalists as well as theorists, often took a leading role. For the theorists this leading role was expressed both through the work on the phenomenology of the standard model and through the introduction of new fundamental concepts. The main milestones in the road beyond the standard model have been grand unified theories, supersymmetry, supergravity and superstrings. CERN played the key role in the development of supersymmetry and very important ones in that of the other three. Although they still remain in the domain of theoretical speculations, they represent thirty years of world effort, thirty years of striving for unity in Nature. I do not believe that they will turn out to be entirely on the wrong track! 
This chapter relates the history of the CERN Theory Division. It is not a review article. Only CERN publications are presented. Occasionally, when a comprehensive report exists on a given subject, I refer to it rather than to the original publications.

\section{References}

[1] A. Peterman, Helv. Phys. Acta 30 (1957) 407.

[2] R. Haag, Dan. Mat. Fys. Medd. 29 (1955) Nr 12.

[3] G. Lüders, Dan. Mat. Fys. Medd. 28 (1954) Nr 5.

[4] L. Michel, Nuovo Cim. 10 (1953) 319.

[5] B. d'Espagnat and J. Prentki, Phys. Rev. 99 (1955) 328.

[6] B. d'Espagnat, J. Prentki and A. Salam, Nucl. Phys. 3 (1957) 446; ibid. 5 (1958) 447.

[7] B. d'Espagnat and J. Prentki, Nucl. Phys. 6 (1958) 596.

[8] B. d'Espagnat and J. Prentki, Nuovo Cim. 24 (1962) 497.

[9] N. Cabibbo, Phys. Lett. 10 (1963) 531.

[10] J.S. Bell and J. Steinberger, Th605, Oxford International Conference on Elementary Particles, Sept. 1965.

[11] G. Zweig, Th401 and Th 412, unpublished.

[12] R. Van Royen and V.F. Weisskopf, Nuovo Cim. 50 (1967) 617; E51 (1967) 583.

[13] S. Fubini and G. Furlan, Physics 1 (1965) 229.

[14] S. Fubini, G. Furlan and C. Rossetti, Nuovo Cim. 40 (1965) 1171.

[15] D. Sutherland, Phys. Lett. 23 (1966) 384; J.S. Bell and D. Sutherland, Nucl. Phys. B4 (1968) 315; M. Veltman, Proc. Roy. Soc. A301 (1967) 107.

[16] J.S. Bell and R. Jackiw, Nuovo Cim. A60 (1969) 47.

[17] J. Wess and B. Zumino, Phys. Lett. 37B (1971) 95.

[18] D.J. Gross and Ch. Llewellyn Smith, Nucl. Phys. B14 (1969) 337.

[19] J. Bros, H. Epstein and V. Glaser, Nuovo Cim. 31 (1964) 1265.

[20] J. Bros, H. Epstein and V. Glaser, Commun. Math. Phys 1 (1965) 240.

[21] A. Martin, 'Scattering Theory: Unitarity, Analyticity and Crossing'. Lecture notes in Physics, Vol. 3, Springer-Verlag, 1969.

[22] J. Bowcock and A. Martin, Nuovo Cim. 14 (1959) 516.

[23] D. Amati, S. Fubini, A. Stanghellini and M. Tonin, Nuovo Cim. 22 (1961) 569; L. Bertocchi, S. Fubini and M. Tonin, Nuovo Cim 25 (1962) 626; D. Amati, A. Stanghellini and S. Fubini, Nuovo Cim 26 (1962) 896.

[24] L. Van Hove, Rev. Mod. Phys. 36 (1964) 655.

[25] L. Van Hove, Phys. Lett. 28B (1969) 429; Nucl. Phys. B9 (1969) 331. For a review see Phys. Rep. 1 (1971) 347.

[26] R. Hagedorn, Nuovo Cim. 15 (1960) 434; Proceedings of the International Conference of Theoretical Aspects of very high Energy Phenomenon, CERN 1961, CERN 61-22, p. 183.

[27] R. Hagedorn, Suppl. Nuovo Cim. 3 (1965) 147; R. Hagedorn and J. Ranft, Suppl. Nuovo Cim. 6 (1968) 169; R. Hagedorn, Astron. and Astroph. 5 (1970) 184.

[28] G. Veneziano, Nuovo Cim. 57A (1968) 190.

[29] V. Glaser, Nuovo Cim. 9 (1958) 990.

[30] H. Epstein and V. Glaser, Lectures at the Les Houches Summer School (1970); Ann. Inst. H. Poincaré 29 (1973) 211.

[31] Th. Kanellopoulos and K. Wildermuth, Nucl. Phys. 7 (1958) 150; see also CERN 59-23.

[32] T.E.O. Ericson and M. Ericson, Ann. Phys. (N.Y.) 36 (1966) 383; for a most recent presentation, see T.E.O. Ericson and W. Weise, 'Pions and Nuclei', International series of Monographs in Physics, 74, Clarendon Press, Oxford 1988.

[33] T.E.O. Ericson, Progr. Part. Nucl. Phys. 1 (1978) 173; M. Ericson and M. Rho, Phys. Rep. 5 (1972) 57; T.E.O. Ericson and W. Weise, Ref. [32], chapter 9. 
[34] J.S. Bell, Rev. Mod. Phys. 38 (1966) 447.

[35] J.S. Bell, Physics 1 (1964) 195; this article, as well as the one of Ref. [34], have been reprinted, together with other articles on the Foundations of Quantum Mechanics, in J.S. Bell, 'Speakable and unspeakable in quantum mechanics' Cambridge University Press, 1987.

[36] M. Veltman, Phys. Rev. Lett. 17 (1966) 553.

[37] J.S. Bell, Nuovo Cim. 50A (1967) 129.

[38] C. Bouchiat, J. Iliopoulos and J. Prentki, Nuovo Cim. 56A (1968) 1150; J. Iliopoulos, Nuovo Cim. 62A (1969) 209.

[39] J. Prentki and B. Zumino, Nucl. Phys. B47 (1972) 99.

[40] Ch. Llewellyn Smith, Phys. Lett. B46 (1973) 233.

[41] H. Leutwyler and J. Stern, Nucl. Phys. B20 (1970) 77.

[42] J. Wess and B. Zumino, Nucl. Phys. B70 (1974) 39.

[43] Ch. Llewellyn Smith and D.V. Nanopoulos, Nucl. Phys. B78 (1974) 205.

[44] W.A. Bardeen, R. Gastmans and B. Lautrup, Nucl. Phys. B46 (1972) 319.

[45] C.H. Albright, Nucl. Phys. B70 (1974) 486; ibid. B75 (1974) 539; C.H. Albright and J. Cleymans, Nucl. Phys. B76 (1974) 48; C.H. Albright and C. Jarlskog, Nucl. Phys. B84 (1975) 467; C.H. Albright, C. Jarlskog and L. Wolfenstein, Nucl. Phys. B84 (1975) 493.

[46] G. 't Hooft and M. Veltman, Th1571, unpublished.

[47] G. 't Hooft and M. Veltman, Ann. Inst. H. Poincaré 20 (1974) 69.

[48] G. 't Hooft, Nucl. Phys. B61 (1973) 455; ibid. B62 (1973) 444.

[49] G. 't Hooft, Nucl. Phys. B72 (1974) 461.

[50] G. 't Hooft, Nucl. Phys. B75 (1974) 461.

[51] G. 't Hooft, Nucl. Phys. B79 (1974) 276.

[52] J. Ellis, M.K. Gaillard and D.V. Nanopoulos, Nucl. Phys. B106 (1976) 292.

[53] E.G. Floratos, D.A. Ross and C.T. Sachrajda, Nucl. Phys. B129 (1977) 66; ibid. B152 (1979) 493.

[54] G. Curci, W. Furmanski and R. Petronzio, Nucl. Phys. B175 (1980) 27; W. Furmanski and R. Petronzio, Phys. Lett. 97B (1980) 437.

[55] H. Grosse and A. Martin, Phys. Rep. 60 (1980) 341.

[56] A.J. Buras, J. Ellis, M.K. Gaillard and D.V. Nanopoulos, Nucl. Phys. 135 (1978) 66.

[57] J. Ellis, M.K. Gaillard and D.V. Nanopoulos, Phys. Lett. 80B (1979) 360.

[58] J. Wess and B. Zumino, Phys. Lett. 49B (1974) 52.

[59] J. Iliopoulos and B. Zumino, Nucl. Phys. 76 (1974) 310.

[60] S. Ferrara, B. Zumino and J. Wess, Phys. Lett. 51B (1974) 239.

[61] J. Wess and B. Zumino, Nucl. Phys. B78 (1974) 1.

[62] S. Ferrara and B. Zumino, Nucl. Phys. B79 (1974) 413.

[63] S. Deser and B. Zumino, Phys. Lett. 62B (1976) 335.

[64] S. Ferrara and P. van Nieuwenhuizen, Phys. Lett. 74B (1978) 333.

[65] S. Ferrara and P. van Nieuwenhuizen, Phys. Lett. 76B (1978) 404.

[66] E. Cremmer, S. Ferrara, C. Kounnas and D.V. Nanopoulos, Phys. Lett. 133B (1983) 61; J. Ellis, A.B. Lahanas, D.V. Nanopoulos and K. Tamvakis, Phys. Lett. 134B (1984) 429; J. Ellis, C. Kounnas and D.V. Nanopoulos, Nucl. Phys. B247 (1984) 373; R. Barbieri, E. Cremmer and S. Ferrara, Phys. Lett. 163B (1985) 143.

[67] K. Bardakci and H. Ruegg, Phys. Lett. 28B (1968) 342.

[68] H.M. Chan, Phys. Lett. 28B (1968) 425; K. Bardakci and H. Ruegg, Phys. Rev. 182 (1969) 1884.

[69] C. Lovelace, Phys. Lett. 32B (1970) 703.

[70] V. Alessandrini, Nuovo Cim. 2A (1971) 321; V. Alessandrini and D. Amati, Nuovo Cim. 4A (1971) 793; V. Alessandrini, D. Amati, M. Le Bellac and D. Olive, Phys. Rep. 1 (1971) 269.

[71] P. Goddard and C.B. Thorn, Phys. Lett. 40B (1972) 235.

[72] D. Olive and J. Scherk, Phys. Lett. 44B (1973) 296.

[73] P. Goddard, C. Rebbi and C.B. Thorn, Nucvo Cim. 12A (1972) 425. 
[74] E. Cremmer and J. Scherk, Nucl. Phys. B50 (1972) 222.

[75] P. Goddard, J. Goldstone, C. Rebbi and C.B. Thorn, Nucl. Phys. B56 (1973) 109.

[76] F. Gliozzi, J. Scherk and D. Olive, Nucl. Phys. B122 (1977) 253. 


\title{
STUDIES IN CERN HISTORY
}

This report is intended for inclusion, in a modified form, in Volume III of the History of CERN. Volume I was published in 1987, and covered the lauching of the European Organization for Nuclear Research. Volume II, concerned with building the laboratory and running it until 1965, was published in 1990. They are available from

\author{
Elsevier Science Publishers \\ Book Order Department \\ P.O. Box 103 \\ 1000 AC Amsterdam \\ The Netherlands
}

The reports in the present series are:

CHS-31 J. Krige, The Relationship Between CERN and its Visitors in the 1970s

CHS-32 J. Krige, Some Socio-Historical Aspects of Multi-institutional Collaborations in High Energy Physics at CERN between 1975 and 1985

CHS-33 A. Russo, The Intersecting Storage Rings: The Construction and Operation of CERN's Second Large Machine and a Survey of its Experimental Programme

CHS-34 I. Gambaro, The Development of Electronic Detectors at CERN (1966-late 1970s)

CHS-35 P.G. Hansen, The SC: ISOLDE and Nuclear Structure

CHS-36 D. Pestre, Gargamelle and BEBC. How Europe's Last Two Giant Bubble Chambers were Chosen

CHS-37 D. Pestre, The Difficult Decision, Taken in the 1960s, to Construct a $3-400 \mathrm{GeV}$ Proton Synchrotron in Europe

CHS-38 K. Winter, Experimental Studies of Weak Interactions

CHS-39 J. Iliopoulos, Physics in the CERN Theory Division

For further information contact J. Krige, History of CERN Project, Building 54, CERN, CH-1211 Geneva 23, Switzerland. 